(Aus der Kgl. Universitäts-Augenklinik zu Bonn.)

\title{
Klinische und pathologisch-anatomische Beiträge zur Kenntnis degenerativer Hornhanterkrankungen.
}

\section{Pannus degenerativus nnd Keratitis bullosa.}

\author{
Von \\ Dr. W. Gilbert, \\ I. Assistenzarzt der Kgl. Universitäts-Augenklinik zu München, \\ früher in Bonn.
}

Mit Taf. I u. II, Fig. 1-9.

Die verschiedenartigen Degenerationsprozesse, die an der Hornhaut erblindeter Augen meist bei vorausgegangener oder noch bestehender Drucksteigerung beobachtet werden, lassen sich im allgemeinen in zwei grössere Gruppen zusammenfassen. Die erste schliesst im wesentlichen in sich die Zerfallserscheinungen an den präexistierenden Gewebsteilen mit sekundären reparativen Vorgängen als direkte Folgen des glaukomatösen Zustandes; die zweite Gruppe umgreift jene klinischen Bilder, als deren Grundlage wir nach dem anatomischen Befunde die Ablagerung von Produkten eines lokal gestörten Stoffwechsels ansehen, also vornehmlich die gürtelförmige Hornhauttrübung und Leukome mit Ablagerung von Kalk und Hyalin. Auch diese Zustände finden sich häufig, aber nicht ausschliesslich an Augen, die infolge von Drucksteigerung oder Iridocyclitis erblindet sind.

Vielfach trifft man pathologische Prozesse, die beiden Gruppen angehören, gemeinsam an, stets aber wiegen Veränderungen der einen Art vor. Jedenfalls bringen es die bei der Bearbeitung der degenerativen Vorgänge in der Hornhaut sich ergebenden Fragen mit sich, das Material nach diesen beiden Gesichtspunkten zu sondern.

Die genannten Prozesse sind nun schon häufig Gegenstand klinischer und histologischer Untersuchungen gewesen. Gleichwohl harren noch manche Fragen, vornehmlich betreffend ihre Genese und ihre gegenseitigen Beziehungen, einer befriedigenden Lösung und zwar in erster Linie, weil vielfach bei genauer Kenntnis des klinischen Bildes die anatomische Untersuchung fehlte, und umgekehrt, 
weil die anatomischen Untersuchungen häufig an Augen angestellt wurden, über die keine genauen klinischen Daten vorlagen.

Das trifft meines Erachtens besonders für die erste Gruppe von $\nabla$ eränderungen zu. Hier handelt es sich um Prozesse, die anscheinend ohne feinere Unterschiede, mehr oder weniger diffus über die ganze Hornhaut ausgebreitet sind, vornehmlich die rauchige Hornhauttrübung, aber auch die Keratitis vesiculosa und bullosa.

Über das Wesen des Anfangsstadiums, der rauchigen Hornhauttrübung, haben uns nun die grundlegenden Arbeiten von Leber (1) und Fuchs $(2,3)$ genügend aufgeklärt, doch es fehlt an einer zusammenfassenden genaueren Bearbeitung der späteren Stadien glaukomatös getrübter Hornhaut. Wohl liegen hinreichend anatomische Einzeluntersuchungen über Keratitis bullosa vor; jedoch man findet über sehr verschiedenartige Veränderungen berichtet und vor allem fehlt es gerade hier an einer vergleichenden Beobachtung klinischer und anatomischer Ergebnisse.

Dank der Anregung meines früheren Chefs und Lehrers, des Herm Geheimrat Saemisch, konnte ich nun eine Reihe von erblindeten und wegen Schmerzhaftigkeit im glaukomatösen Stadium enucleierten Augen anatomisch untersuchen, von denen mir grösstenteils selbstgefertigte genaue klinische Notizen vorlagen, während von einigen andern mir sorgfältig geführte Krankengeschichten und vor allem übersichtliche Skizzen des klinischen Bildes zur Verfügung standen, so dass eine gleichermassen die klinischen und anatomischen Verhältnisse berücksichtigende Untersuchung ermöglicht wurde.

In vorliegender Arbeit sind nun die Befunde, welche die Veränderungen der ersten Gruppe umfassen, zusammengestellt, während die Untersuchung einer weiteren Reihe von Augen, die der zweiten Gruppe zugehören, einer späteren Mitteilung vorbehalten sei.

Fall I.

Johann D. trat zum erstenmal im Jahre 1885 in die Behandlung der Bonner Universitätsaugenklinik; er gab an, im Herbst 1884 sei ihm ein Stahlsplitter gegen das linke Ange geflogen; dieses sei einige Tage entzündet gewesen, aber ohne irgend welche Behandlung wieder völlig ausgeheilt. Etwa 5-6 Monate nach dieser Verletzung trat Sebstörung ein, die durch Glaskörpertrübungen bedingt war. Nach kurzer klinischer Behandlung wurde Patient mit voller Sehschärfe entlassen; irgend welche Narbe, die für eine perforierende Verletzung hätte sprechen können, wurde damals nicht beobachtet. Im Verlaufe der vächsten Jahre trat aber wieder allmählich Abnahme des Sehvermögens ein, so dass 1888 auswärts Iridektomie und Extraktion gemacht wurde. Erst im Jahre 1899 
traten wiederum Entzündungserscheinungen and Abnahme des Sehvermögens ein; schon damals bestand glaukomatöser Habitus, rauchige Hornhauttrubung, Excavation der Papilla nervi optici und Drucksteigerung. Vis. c. $3 / 60+10,0 D$. 1902 erblindete das Auge völlig.

20. V. 1905. Wiederaufnahme des jetzt 47 Jahre alten Patienten wegen häufig wiederkehrender Schmerzanfalle.

Die Oberfläche der Hornhaut des linken Auges ist mit Ausnahme eines schmalen oberen Randsanmes leicht uneben, das Epithel matt, gestippt. Etwas unterhalb und einwärts vom Hornhautzentrum findet sich im vorderen Parenchym ein kleines, rundes, graugelbliches Infiltrat, über dem das Epithel grossenteils sich abgestossen hat. Der temporale Hornhautrand ist grauweisslich infiltriert, zeigt aber keine Gefässe. Die vordere Kammer ist vertieft (Aphakie); die Iris zeigt abgesehen vom Colobom keine Besonderbeiten. Trübroter, in der Gegend der Papille weisser Reflex. $T+2$. Amaurose.

22. V. Unterhalb und nasal vom Zentrum hat sich über dem Infiltrat eine grosse, schwappende Blase, in der Umgebung noch mehrere kleinste Bläschen gebildet. Diese Blase ist am 23. V. geplatzt, worauf die Schmerzen vorübergehend bis zur Bildung einer nenen Blase aussetzen.

28. $\dot{V}$. Enucleatio bulbi.

Nach Fixation in Formalin und Nachbärtung in Alkohol wird der Bulbus vertikal aufgeschnitten so, dass die grössere temporale Hälfte den ganzen Sehnerv und etwa $3 / 5$ der Hornhaut enthält. Diese grössere Bulbushälfte wird in Celloidin eingebettet and in Schnitte von 20-30 $\mu$ Dicke zerlegt; jeder zweite Schnitt wird untersucht. Die kleinere nasale Hälfte der Cornea wird in Paraffin eingebettet und die ganze lückenlose Schnittserie von $8 \mu$ Dicke durchgefärbt.

\section{Mikroskopischer Befund.}

Beginnen wir die Untersuchung der ganzen vertikalen Schnittserie im temporalen Limbus, so finden wir unten etwa $3 \mathrm{~mm}$ hinter and unterhalb der letzten Ciliarkörperfalte eine die Sklera und Chorioidea schräg von vorn nach hinten durchsetzende Narbe. Die Pars plana corporis ciliaris ist an den Rändern der Narbe gleichsam scharf abgeschnitten; an ihrer Stelle findet man ein Schwartengewebe, welches in den Glaskörperraum $0,5 \mathrm{~mm}$ hineinprominiert und an den Rändern der Narbe etwas über die Pars ciliaris retinae hinübergreift. Als nach Feststellung dieser klinisch nicht sichtbar gewesenen Narbe die Färbung auf eisenhaltiges Pigment nach Perls vorgenommen wird, färben sich die Ciliarepithelien, besonders die Firsten der Proc. ciliares in der Nachbarschaft der Narbe lebhaft blau.

Die Kammerbucht ist oben gut erhalten und frei von Verwachsungen; nur unten ist sie stellenweise peripher aufgehoben, indem die Wurzel der Irisschenkel seitlich vom Colobom der Hornhauthinterfläche anliegt. Ansammlnngen von Eisenkörnchen sind in der Kammerbucht und dem angrenzenden Gewebe nicht zu sehen. Im Balkenwerke zwischen Kammerwinkel und Schlemmschem Kanal sieht man vielfach Rundzellenansammlungen. Der Sehlemmsche Kanal ist fast überall ganz verödet. 
Die Lumina der episkleralen Gefässe sind weit entfaltet. In den mittleren Schichten der Sklera finden sich mehrfach kleinzellige Infiltrationsstränge. Diese Infiltration setzt sich auch auf die temporale Randzone der Cornea fort und zwar zieht ein rundzellenführender Strang vom oberen Limbus durch die mittleren Corneallamellen zum unteren Limbus. Diese kleinzellige Infiltration der Cornea nimmt schnell ab, sobald sich die Schnitte etwas mehr vom Limbus entfernen, reicht aber am weitesten (etwa $300-400 \mu$ vom Limbus) in die untere Cornealhälfte hinein; kleinere Ansammlungen von Rundzellen finden sich in der ganzen temporalen Cornealhälfte und nehmen nasalwärts immer mehr ab. Ödem des Hornhautparenchyms ist nur im geringen Grade vorhanden.

Im Limbus begegnet man des weiteren temporal unten auf der Grenze von Episklera und vordersten Sklerallamellen einer Anhäufung tropfen- and schollenförmiger Gebilde, hyaliner Konkremente, auf die genauer an anderer Stelle eingegangen werden soll.

Zwischen den Konkrementen und dem Conjunctivalepithel findet sich ein Gewebe fibrillärer Struktur, das reich an Bindegewebskernen und Leukocyten ist, mit Eosin und Säurefuchsin sich aber viel weniger intensiv färbt als Corneal- und Sklerallamellen. Einzelne Gefässquerschnitte sieht man nur in der Nähe der Konkremente. Dies fibrilläre Bindegewebe setzt sich ohne Unterbrechung und ohne Veränderung seiner Struktur vom unteren Limbus auf die Cornea fort, zwischen Epithel und Bowmanscher Membran verlaufend. Diese subepitheliale Gewebsschicht uberzieht unter mannigfachen Veränderungen ihres Volumens und ihrer Struktar die ganze Cornea, indem nur an ganz vareinzelten Stellen Epithel und Bowmansche Membran einander unmittelbar anliegen. Am kräftigsten zeigt sich das Gewebe temporal in der unteren Cornealhälfte entwickelt, wo es $1 / 4-1 / 3$ der Cornea propria erreicht und wiederum ein wenig temporal vom Cormealzentrum, wo es stellenweise $1 / 3$ bis nabezu die Hälfte der Dicke des Cornealparenchyms besitzt; im übrigen ist die Stärke dieser Gewebslage sehr variabel und stellenweise ist sie bis auf die Dicke der vorderen elastischen Lamelle und noch weniger reduziert; im allgemeinen aber ist die Schicht in der unteren Cornealhälfte stets kräftiger entwickelt als in der oberen, wo sie meist als schmaler Strang zum Limbus zieht.

Am temporalen Cornealrand hat dieses Gewebe den typischen Bau eines fibrillären, anscheinend völlig gefässfreien Bindegewebes (vgl. Taf. I, Fig. 1); es ähnelt, abgesehen von dem oben schon hervorgehobenen Unterschied in der Färbbarkeit, ziemlich ausgesprochen dem Cornealparenchym, von dem es aber bis auf eine scharf umschriebene Stelle überall durch die intakte vordere Grenzlamelle getrennt ist. Von der Cornealgrundsubstanz unterscheidet es sich ferner durch einen bedentend grösseren Reichtum an eingestrenten Leukocyten. Diese liegen nun bisweilen zwischen den Gewebsfasern mit ihrer Längsseite zu mehreren, 3, 4, 5 and noch mehr perlschnurartig hintereinandergereiht, wobei zwischen den einzelnen Lenkocyten nur ganz kurze, ungefärbte Zwischenrăume bleiben; sie scheinen also in feinen Kapillaren zu liegen, deren Lumen von ihnen ganz erfüllt wird, daher nicht sichtbar ist. 
Das Cornealepithel zeigt in dieser temporalen Randzone nur wenig Abweichungen von der Norm; es liegt unmittelbar der Bindegewebsschicht auf und besteht aus fünf Lagen von regelmässigem Bau; doch begegnet man auch hier schon einzelnen Partien, wo die Bindegewebsschicht etwas stärker, das Epithel auf drei Zellreihen mit Verwischung der histologischen Differenzierung zwischen Basalzellen und denen der Mittellage reduziert ist; hier und da treibt das Epithel auch schon sporn- and leistenartige Fortsätze in die Bindegewebslage hinein (Taf. I, Fig. 1 u. 2). Schon $0,5 \mathrm{~mm}$ vom Limbus rücken die letztgenannten Veränderungen im Epithel mehr in den Vordergrund und verändern das histologische Bild erheblich und zwar spielen sich die folgend beschriebenen Prozesse vorwiegend in der Umgebung des horizontalen Meridianes ab. Bindegewebsschicht und Epithel streiten hier gewissermassen um den Raum. Die erstere treibt papillenähnliche Fortsätze gegen das Epithel, welches so von der Rückseite her wie angenagt anssieht (Taf. I, Fig. 1). Das Epithel seinerseits füllt jede Lücke zwischen den Bindegewebsfibrillen aus und sendet zungen- und spornartige Ausläufer zwischen die Hervorragungen des Bindegewebes in die Tiefe, so dass man anch stellenweise freie Epithelperlen im pannösen Gewebe antrifft. Die in das Epithel hineinragenden Bindegew ebssprossen unterscheiden sich im Bau und auch tinktoriell ziemlich erheblich von den tieferen Bindegewebslagen. Erstere sind nämlich erbeblich ärmer an fixen Zellen und auch an eingestrenten Lenkocyten (Taf. I, Fig. 1). Da sie auch die Protoplasmafarbstoffe sehr wenig annehmen, ist die fibrilläre Struktur schwer erkeunbar, am deutlichsten noch an der $\mathrm{Ab}$ grenzung gegen das Epithel hin, die durch eine den Vorragungen mäanderbandartig folgende Gewebsfibrille von annähernd der Dicke der Bowmanschen Membran gebildet wird. Die Dicke der Epithelschicht, die Zahl der Zellreihen schwankt tiber diesen Partien sehr erheblich. $Z$ wei- und dreireihige Partien wechseln mit solchen von vier, sechs, ja sieben bis acht Reihen ständig ab. Eine basale Cylinderzellenschicht ist wohl als solehe noch an den meisten Stellen erkennbar; vielfach sind aber die Unterschiede zwischen Basalzellen und den polygonalen der mittleren Schichten verwischt. Wo die Basalzellen deutlich den Charakter von Cylinderepithelien aufweisen, da begegnet man auch hier und da Kernteilungsfiguren.

Weitere auffallige Epithelveränderungen finden sich in den nun folgenden Schnitten, welche durch das Gebiet führen, wo in der unteren Cornealhälfte rezidivierende Blaseneruption beobachtet worden war. Hier liegt auf der Grenze vom mittleren zum unteren Cornealdritiel in einer wechselnden Ausdehnung von 2,5-3,0 mm (Vertikalschnitte) das Epithel der vorderen Grenzlamelle unmittelbar auf (Taf. II, Fig. 3). Diese von subepithelialem Bindegewebe freie Stelle erstreckt sich im horizontalen Durchmesser ungefähr bis zur Mitte der Cornea. Eine eigentliche Schichtung in Zellagen vermisst man hier im Epithel, vielmehr handelt es sich um regellos neben- und übereinander gelagerte Zellen von durchweg polygonaler Form. Die Zellen der obersten Lage sind etwas abgeflacht; die basalen, der Bowmanschen Membran aufliegenden Zellen tragen hier nirgends den Charakter von Cylinderepithelien. Hier und da heben sich 
einige Zellgruppen aus dem regellosen Nebeneinander durch eine konzentrische Anordnung heraus, so dass ein den Carcinomperlen etwas ähnelndes Bild entsteht. Am temporalen Rand dieser von subepithelialem Bindegewebe freien Zone übertrifft diese neugebildete Epitheldecke die normale stellenweise um das doppelte an Dicke und Zellreihenzahl; weiter nasal verdünnt sich die Epitheldecke immer mehr, bis sie auf zwei, ja nur mehr eine Zellage reduziert ist.

Hier, an der Stelle, wo klinisch ein Infiltrat beobachtet worden war, finden sich nun auch an der Bowmansehen Membran und an der Cornea propria pathologische Prozesse. Das Cornealparenchym ist hier zunächst nicht unerheblich verdünnt. In den Schnitten, die noch ausserhalb des Infiltrates liegen, sind die Corneallamellen zwar noch völlig frei von Infiltration; aber das Fehlen der Bowmanschen Membran und zwei kleine narbige Einziehungen der vorderen Lamellen (Taf. II, Fig. 3) zeigen an, dass auch hier im vorderen Parenchym sich entzündliche Veränderangen früher abgespielt haben. Auch auf den nächsten weiter nasal gelegenen Schnitten, wo sich nur eine einreihige Epithellage vorfindet, fehlt die Bowmansche Membran, welche oben und unten, allmählich sich zuspitzend, aufhört. Die obersten Corneallamellen sind hier zerstört, die tieferen Schichten bis beinahe zur Mitte des Cornealparenchyms kleinzellig infiltriert; hier fehlt ausser der Grenzlamelle auch die Epithelbedeckung auf eine kurze Strecke, ungefähr 0,5 qmm, völlig.

Die Epithelzipfel, die von oben und unten zu dem kleinen Defekt ziehen, enthalten zwischen grossen polygonalen Zellen mit gut gefärbtem Kern zahlreiche kleinere und grössere farblose, stark lichtbrechende, blasige Hohlräume von runder, ovaler und auch langgestreckter Form, die in der Grösse 1-2 und mehr Epithelien gleichkommen. Fast stets handelt es sich um tropfenartige, völlig homogene Gebilde; nur vereinzelt sieht man noch Reste von Protoplasmafäden oder Zellgrenzen in das Lumen hineinragen. Mehrfach finden sich in einem grösseren solehen Gebilde 2-4 sehr dunkel tingierte Zellkerne, die aber bis auf Bruchteile normaler Epithelkerne gesehrumpft sind und keinen Protoplasmahof mehr erkennen lassen; auch sieht man in der Umgebung solcher Hohlräume zuweilen einige dunkle kleine Körnchen (Kerntrümmer?). Auch bei Anwendung der Färbemethoden zum Nachweise des Fibrins, der schleimigen und hyalinen Degeneration bleiben diese tröpfchenartigen Gebilde ungefärbt. Auch auf Schnitten, die noch weiter nasal, durch den nasalen Rand des Infiltrates gehen, wo die Epithellage wieder ununterbrochen die ganze Cornea mehrschichtig überdeckt, finden sich zerstreut solche blasige Gebilde von anscheinend flüssigem Inhalt zwischen den Basalzellen, mehrfach ist aber auch eine Reihe von 4-6 Basalzellen nebeneinander zugrunde gegangen und völlig ausgefallen, so dass an ihrer Stelle sich ein leerer Hohlraum findet, in den die basalen Enden der Zellen der zweiten Epithelreihe mit feinen Ausläufern hineinragen. Fast überall, wo diese Epithelveränderungen vorliegen, da ist anch die Bowmansche Membran auf kurze Strecken völlig unterbrochen.

Bei der Verfolgung dieser Epithelveränderungen sind wir schon bis an den nasalen Rand des Pupillargebietes gekommen. Wenden wir uns 
nun zur Untersuchung des Pannus zurück, so finden wir noch temporal vom Pupillargebiet auf Schnitten, in denen auch der Musc. sphincter iridis gelegen ist, neue bemerkenswerte Eigentümlichkeiten seiner Struktur. Die bindegewebige Schicht nämlich, die mit zunehmender Entfernung vom temporalen Limbus allmählich immer kernarmer geworden ist, wird über dem temporalen Teil der Pupille immer mehr homogen und verliert ihre fibrilläre Struktur, bis man schliesslich über der Mitte des Pupillargebietes zwischen Epithel und Bowmanscher Membran eine ganz kernfreie, homogene, anscheinend strukturlose und gleichsam gallertig aussehende Masse vor sich hat (Taf. I, Fig. 2), in die das Epithel auch hier sporn- und leistenartige Fortsätze hineintreibt. Mit Eosin und mit Säurefuchsin färbt sich diese homogene Schicht etwas schwächer als die Hornhautgrundsubstanz; Carminfarbstoffe nimmt sie überhaupt kaum an. Bei Anwendung aller dieser Färbemethoden erscheint dies Gewebe völlig homogen, und auch unter Benutzung starker Trockensysteme ist keine Struktur zu erkennen. Erst bei Anwendung der neueren spezifischen Bindegewebsfärbemethoden nach Mallory-Ribbert und nacb E. Fränkel ${ }^{1}$ ), bei denen dies Gewebe den für Bindegewebe charakteristischen Farbton annimmt, gelingt es hier und da, einzelne Gewebsfbrillen zu Gesicht zu bekommen. Bei genauester Durchsicht sämtlicher Präparate findet man in dieser homogenen Schicht doch noch vereinzelt hier und da eingestreut Leukocyten und auch schwächer tingierte, langgestreckte, spindelige Bindegewebszellen. In vielen Schnitten lässt sich auch der kontinuierliche Übergang dieser homogenen Schicht in ein Gewebe von deutlich erkennbarer fibrillärer Struktur verfolgen (siehe auch Taf. I, Fig. 1).

Eine weitere interessante histologische Einzelheit beobachtet man vornehmlich, wenn auch nicht ausschliesslich dort, wo in der Nachbarschaft des Epitheldefektes, bzw. der Epithelverdünnung lebhafte Epithelneubildung stattgefunden hat, wo sich unter die Reste der alten Epitheldecke - kenntlich an deren basaler Cylinderzellenlage - neu gewuchertes Epithel geschoben hat (Taf. I, Fig. 4). Zwischen diesen beiden Epithellagen findet man mehrfach feine Gewebsbänder ungefähr von der Dicke der Bowmanschen Membran, die von der subepithelialen Gewebslage abzweigen, zwischen beiden Epithellagen durch das Epithel bandartig ziehen und entweder frei im Epithel endigen, oder, was häufiger der Fall ist, sich weiterhin wieder der Bowmanschen Membran oder der pannösen Schicht auflegen. Diese Erscheinung beobachtet man hauptsächlich an der Stelle, wo klinisch und anatomisch Blasenbildung vorlag, wo die pannöse Lage nur ganz schwach entwickelt ist. Diese Gewebsbänder stellen somit Ausläufer der pannösen Schicht in das vom Pannus noch frei gebliebene Gebiet uber dem Infiltrat im Cornealparenchym dar. Vereinzelt werden diese mitten durch das Epithel ziehenden Bänder aber auch in der oberen Cornealhälfte gefunden (Taf. II, Fig. 3). Stets verläuft dann uber dem Bindegewebsband eine zwei- bis dreireihige Epithelzellenschicht, deren unterste meist cylindrischen Bau aufweist, während die mittlere

1) Schmorl, Untersuchungsmethoden, 3. Aufl, S. 121. 
kabische, die obere abgeflachte Zellen enthält. Die Epithelien unter dem Bande sind kubiseh und fast durchweg durch eine schmale Bindegewebsschicht von der Bowmanschen Membran getrennt. Ein Teil dieser intraepithelialen Gewebsbänder ist kernbaltig (Taf. I, Fig. 4) und zwar kann man wieder deutlich Leukocyten und Bindegewebszellkerne unterscheiden. In den Schnitten, in denen der Pannus homogen und strukturlos erscheint, da verlaufen auch die Bänder als völlig kernfreie, homogene, anscheinend ganz strukturlose, blassgefärbte Gewebsstreifen intra- und subepithelial (Taf. I, Fig. 5).

Die Epithelien unter den intraepithelialen Bändern gehen stellenweise auch degenerative Veränderungen der gleichen Art ein, wie sie oben von den Epithelien über dem parenchymatösen Hornhautinfiltrat schou beschrieben sind. Der protoplasmatische Zelleib ist $\mathrm{zu}$ einer glasigen, stark lichtbrechenden Masse aufgequollen, in der der geschrumpfte and sehr stark tingierte Zellkern meist randständig liegt, während vom Zellgerüst kanm mehr etwas wahrzunehmen ist. Diese Epithelveränderungen finden sich aber nur in der nasalen Hälfte der Cornea, wo die subepitheliale Schicht ubberhaupt viel schwächer entwickelt ist und auch hier nur dort, wo sie bis auf Bruchteile der Dicke der Bowmanschen Membran reduziert ist oder gar ganz fehlt. Letztores trifft für die dem nasalen Hornhautviertel angehörenden Schnitte in der Umgebung des horizontalen Durchmessers mehrfach $\mathrm{zu}$, während auch hier am Limbus ubberall sich eine straffe Lage fibrillären Bindegewebes vorfindet.

Aus dem übrigen anatomischen Befunde sei nur hervorgehoben, dass die Retina hinter der Ora serrata nur geringe Siderosis zeigt und total atrophisch ist. Die Papilla nervi optici ist hochgradig steilwandig excaviert.

Im Intervaginalraum finden sich, teils seiner äusseren Wand, der Duralscheide anliegend, teils im Netzwerk der Arachnoidea zahlreiche, geschichtete Konkremente, wie sie in senilen Augen häufig gefunden werden. Sie färben sich sehr stark mit Hämatoxylin, die Farbreaktionen des Hyalin and des Amyloid geben sie nicht ${ }^{1}$ ).

Fassen wir das Ergebnis der anatomischen Untersuchung zusammen, so hat sie zunächst 21 Jahre nach der Verletzung den einwandfreien Nachweis erbracht, dass ein Eisensplitter bei jener anscheinend so unbedeutenden Verletzung damals in das Auge gedrungen war. Zieht man in Erwägung, dass jene Verletzung ursprünglich nur einen sehr geringfügigen, spontan abklingenden Reizzustand zur Folge gehabt hat, dass ferner die Siderosis nur auf eine circumscripte Stelle, auf die Epithelien der Pars ciliaris retinae in der Umgebung der Eingangswunde beschränkt war, so erscheint die Annahme berechtigt, dass es sich um einen sehr kleinen Eisensplitter gehandelt hat. Es darf daher nicht wundernehmen, dass der Fremdkörper

1) Präparate von Fall I wurden bei der 34. Versammlung der ophthalmologischen Gesellschaft zu Heidelberg 1907 demonstriert. Siehe den Ber. S. 290. 
bei der Sektion des Augapfels nicht gefunden worden ist, zumal ein solcher nicht im Innern des Auges vermutet worden war. Vielmehr darf mit Sicherheit angenommen werden, dass der kleine Splitter im Verlauf der 20 Jahre völlig aufgelöst worden ist.

Diese Annahme würde auch die Entstehung des Glaukoms im aphakischen Auge bei grösstenteils gut erhaltener Vorderkammer verständlich erscheinen lassen. Ist es doch Erdmann(4) gelungen, durch elektrolytisch dargestelltes feinkörniges Eisen experimentelles Glaukom zu erregen. Zwar bestehen zwischen Erdmanns anatomischen Befunden und dem hier vorliegenden erhebliche Differenzen, die sich aber durch die wesentlich andern Cirkulations- und Resorptionsverhältnisse beim Tierexperiment erklären. Bei Erdmann ist das Glaukom nämlich die Folge einer Verlegung der vorderen Abflusswege durch Obliteration des Kammerwinkels, welche durch eine proliferierende Entzündung im Filterwerk des Kammerwinkels zu stande kommt. Bei diesen Tierversuchen wird plötzlich eine grosse Menge elektrolytisch dargestellten Eisens in die Kammer gebracht, und proliferierende Entzündung mit Verlegung des Kammerwinkels ist die notwendige Folge. Anders nach Erdmann schon bei kurzdauernder Elektrolyse und spärlicher Eisenabscheidung. Hier gehen „die Entzündungserscheinungen in der Regel in wenigen Tagen zurück, und das Auge erlangt seine normale Beschaffenheit wieder, indem die Eisenmassen teils resorbiert werden, teils freiliegend oder in Zellen eingeschlossen einheilen". In Lösung befindliches Eisen filtriert in die ableitenden Venen. Bei dem schnellen Ablauf der Versuche kommt es auch nicht zur Siderosis. In unserem Falle hingegen wird die chemische Lösung des Eisens durch die Körpersäfte sich so langsam vollzogen haben, dass die jeweils zur Filtration kommenden Mengen resorbiert werden konnten, ohne dass es zur proliferierenden Entzündung und zur Obliteration des Kammerwinkels kam. Erst ganz allmählich im Laufe der Jahre beginnen die Abflusswege zu versagen - Verödung des Schlemmschen Kanals und nun kommt es auch zu Rundzellansammlung in der Umgebung des ableitenden Plexus. Da nur wenige Schnitte für die Eisenreaktion verwertet werden konnten, liegt auch kein Widerspruch gegen diese Auffassung in der Tatsache, dass im Kammerwinkel keine Eisenteilchen nachgewiesen werden konnten. So könnte diese Beobachtung wohl ein klinisches Analogon zu Erdmanns Experimenten darstellen.

Soviel uiber die vermutliche Genese dieses Glaukoms. Unter dem Einfluss der dauernden Drucksteigerung ist es nun zu einer 
Erkrankung des Hornhautepithels gekommen, welche zur Zeit der Untersuchung vornehmlich an circumscripter. Stelle in der unteren nasalen Hornhautpartie nachzuweisen war und klinisch den Charakter der Keratitis bullos a trug. Hier spielte sich auch unter der auf kurze Strecke zugrunde gegangenen vorderen Grenzlamelle im vorderen Parenchym ein Entzündungsprozess ab; ob dieser ektogen oder endogen entstanden ist, lässt sich aus dem mikroskopischen Bilde kaum entscheiden; wahrscheinlich aber - und hierfür spricht auch die klinische Beobachtung - hat es sich ursprünglich um ein parenchymatöses Infiltrat gehandelt, als dessen Folge sich Blasenbildung im Epithel eingestellt hat. Auf diesen Zusammenhang hat bekanntlich Saemisch(5) zuerst hingewiesen. Die Genese des Infiltrats wäre dann vielleicht so $\mathrm{zu}$ denken, dass die Anhäufung hyaliner Schollen im unteren Limbus einen Entziundung erregenden Reiz ausgelöst hat; äussert doch auch $\mathrm{Fuch}$ ( $(6)$ die Meinung, dass die Anhäufung hyaliner Schollen vielleicht einen Reiz setze, welcher das Auswachsen von Gefässen veranlasst. Die entzündliche Infiltration hätte alsdann nach Zerstörung der Bowmanschen Membran durch die stets erneute Durchtränkung mit Ödemflüssigkeit zu degenerativen Prozessen im Epithel geführt. Da diese Veränderungen hinter der Pannusbildung. erheblich zurücktreten, mögen sie im wesentlichen erst mit den folgenden Fällen von Keratitis bullosa abgehandelt werden und sollen hier zunächst nur insoweit Berücksichtigung finden, als zum Verständnis des anatomischen Gesamtbildes erforderlich ist.

Das bemerkenswerteste Resultat der Untersuchung ist nämlich der anatomische Nachweis einer klinisch nicht diagnostizierten subepithelialen Gewebsschicht, welche, im einzelnen vielfache Abweichungen im Bau zeigend, fast kontinuierlich die ganze Cornea über der Bowmanschen Membran überzieht und ihre Entwicklung anscheinend vorwiegend vom temporalen Limbus her genommen hat. Die hier vielfach perlschnurartig hintereinander gereihten Leukocyten zwischen den Gewebsfibrillen deuten darauf hin, dass hier noch Kapillaren vorhanden sind, wenngleich anatomisch Gefässlumina nicht mehr nachgewiesen werden können. Hier am temporalen Limbus hat diese Gewebslage auch offenbar dank ihrem Zellreichtum zur grauweisslichen Verfärbung des Limbus geführt.

Der weiteren Würdigung des anatomischen Befundes sei nun ein kurzer Hinweis auf die einschlägigen bis heute vorliegenden Untersuchungen vorausgeschickt. Nachdem H. Müller(7), Donders (8), Althof(9), Iwanoff(10), Pagenstecher und Genth(11) u. A. an 
erblindeten Augen zwischen Hornhautepithel und Bowm an scher Membran schon „eine ziemlich gleichmässige, membranöse Schicht" (H. Müller), „eine Lage faserigen Gewebes" (Donders) beschrieben batten, gebührt Baas (12) das Verdienst, scharf diesen an degenerierten glaukomatösen und iridocyclitischen Augen beobachteten Pannus vom skrofulösen und trachomatösen Pannus getrennt und ihm die Sonderstellung zugewiesen zu haben, die ihm schon nach seiner Genese zukommt. Es ist daher nicht mehr statthaft, die Befunde von Pannus zu verallgemeinern und etwa, wie es früher wohl geschehen ist, von einem Pannus an einem degenerierten Auge Schlüsse auf die Anatomie des Pannus skrofulosus oder trachomatosus zu ziehen oder umgekehrt. Vielmehr entspricht der andern Entstehungsweise des Pannus bei den verschiedenen klinischen Bildern auch ein anderes anatomisches Substrat; und zwar ergibt der übereinstimmende Befund auch der neueren Untersucher [Baas (12), Hertel (13), A u g stein (14), Seo and Yamaguchi (15)], sowie Ushikubo (48), denen Verfasser sich auf Grund eigener Untersuchungen an Leichenaugen anschliessen möchte, dass der skrofulöse Pannus sich primär unter der Bowmanschen Membran verbreitet, wie es auch bei der konstitutionellen Art des Grundleidens nicht anders zu erwarten ist. Für den Pannus trachomatosus ist das anatomische Bild noch nicht fest umschrieben. Stehen doch hier den Angaben von Ritter (16), Raehlmann(17), Michel (18), Junius(19) und Greeff(20), dass beim Trachom die entzindliche Infiltration sich unter der anfänglich noch vorhandenen Bow man schen Membran entwickelt, die Befunde von Fuchs(21) und Bietti(22) entgegen. Diese Autoren fanden nämlich beim Trachom den Pannus auch subepithelial ïber der Bowmanschen Membran. Bietti nimmt daher an, dass der trachomatöse Pannus sich sowohl über wie unter der Bowmanschen Membran entwickeln kann; dieser Ansicht schliesst sich auch de Vincentiis (23) an. Der degenerative Pannus findet sich dagegen primär ausnahmslos zwischen Epithel und Bowm an scher Membran [Baas, Greeff, Ginsberg (24)], nur nach Läsion der Bow manschen Membran stellenweise auch unter dieser[Meller(49)]. Nach Baas ist das frühe Stadium durch reichliche rundzellige Infiltration, viele Gefässe und noch nicht sehr viel neugebildetes Bindegewebe gekennzeichnet. Im älteren Narbenstadium finden sich dicke sklerotische Bindegewebsauflagerungen auf der Bow manschen Membran. Er erfährt also dieselbe bindegewebige Umwandlung, die Fuchs für den älteren Pannus trachomatosus beschreibt, indem ein Teil der Zellen, aus welchen der Pannus besteht, zu Spindelzellen und endlich zu 
Bindegewebsfasern auswächst. Weiteren Aufschluss über die Histologie des Pannus degenerativus erbrachte sodann Foster(25), der darauf hinwies, dass das Gewebe später oft homogen, „hyalinartig" wird, so dass keine Zellen mehr zu erkennen sind $^{1}$ ). Die gleiche Beobachtung eines zell- und gefässarmen Gewebes unter dem Epithel findet sich übrigens schon in mehreren Fällen Althofs.

Hier setzt nun die Bedeutung vorliegenden Falles ein, der wohl geeignet erscheint, unsere klinischen und anatomischen Kenntnisse vom Pannus degenerativus etwas zu erweitern.

Die Präparate vom temporalen Hornhautdrittel zeigen nämlich überall, die übrigen stets zum mindesten in der Nähe des Limbus einen so deutlich bindegewebigen Bau, dass an der Diagnose „Pannus degenerativus im älteren Narbenstadium" nicht wohl gezweifelt werden kann. Über den mittleren Teilen der Cornea geht nun aber das Gewebe eine erhebliche Veränderung ein, indem es die fibrilläre Struktur immer mehr verliert, immer zellärmer und homogener wird. Bei Untersuchung eines derartigen mit Hämatoxylin-Eosin gefärbten Schnittes ist man versucht, zu glauben, es handle sich um hyaline Umwandlung oder um eine Lage geronnener Flüssigkeit. Hyaline Entartung des Gewebes liegt nun nicht vor, denn diese Gewebslage gibt nicht die charakteristischen Färbereaktionen des Hyalins und verhält sich auch chemisch anders: es wird durch Säuren und Alkalien zerstört. Auch um geronnene Flüssigkeit, wie sie bei Keratitis bullosa ja vielfach beobachtet sein soll, kann es sich nicht handeln, da die Fibrinfärbungen negativ ausfallen; ebenso ergeben die üblichen Methoden zum Nachweis schleimiger Entartung ein negatives Resultat. Somit gewinnt schon per exclusionem die Annahme, dass es sich um Bindegewebe handle, viel an Wahrscheinlichleit. Aber der anatomische Befund selbst bietet noch mehrere direkte Anhaltspunkte hierfür. Denn einmal kann man mehrfach den Übergang dieser strukturlosen Schicht in Gewebe von fibrillärer Struktur verfolgen, sodann triff man doch noch ganz vereinzelt in dieser homogenen Schicht zellige Elemente an und zwar vorwiegend Rundzellen, in der tieferen Lage aber, direkt über der Bowmanschen Membran, auch Bindegewebszellen. Zudem verhält sich dies Gewebe auch tinktoriell wie Bindegewebe, wie vor allem die eleganten elektiven Färbemethoden von Mallory-Ribbert und E. Fränkel schlagend beweisen.

1) Vgl. die Taf. I, Fig. 2 und Ginsberg, Fig. 33 . 
Diese etwas umständliche Beweisführung erscheint deshalb notwendig, weil, wie ich glaube, früher schon häufiger solche homogene Gewebslage bei Keratitis bullosa gesehen, aber für geronnene Flüssigkeit gehalten worden ist.

Manche der frei im Epithel endigenden Membranen (siehe S. 7) zwängen sich derart zwischen die Reste der alten und die neue Epitheldecke ein, dass man wohl geneigt sein könnte, sie in genetische Beziehung zu-den unter ihnen liegenden degenerierenden Epithelien zu bringen, sie also als cuticulare Ausscheidungsprodukte zu betrachten nach Art der Bildung des endothelogenen Bindegewebes von seiten des Hornhautendothels. Sehen wir daron ab, dass die Bildung solcher cuticularer Abscheidungen von seiten des Hornhautepithels - wenigstens meines Wissens - bisher nicht bekannt ist, dass weiter die Bildung glashäutiger Membranen in der Vorderkammer aufReizung, nicht aber auf Degeneration des Hornhautendothels erfolgt, so ergibt wieder der Ausfall der spezifischen Färbemethoden, dass wir nicht epitheliale Abkömmlinge, sondern ein Gewebe collagener Natur vor uns haben.

Als die mikroskopische Untersuchung mit den Vertikalschnitten der Hornhautmitte begonnen wurde, deutete auch ich die subepitheliale homogene Schicht als Lage geronnener Flüssigkeit und die homogenen Bänder als strukturlose, accessorische Membran [Fuchs (3), Wirths (26)]. Erst die systematische Untersuchung der ganzen Cornea lieferte den sicheren Nachweis, dass es sich um Bindegewebe (sklerotisches, homogenes, "hyalines", wie es früher auch schon benannt worden ist) handelt, welches durch ungünstige Verhältnisse mechanischer Art - dauernde Drucksteigerung bei wiederholtem Abstossen der Epitheldecke - sowie nutritiver Art —, mangelhafte Ernährung von seiten der nur in der Nähe des temporalen Limbus vorhandenen spärlichen Gefässe - sklerotisch, homogen geworden war. Übrigens beschreiben auch de Schweinitz und Shumway (27) eine von der äussersten Peripherie sich zwischen Bowmansche Membran und Epithel erstreckende 7-9 $\mu$ dicke Membran von homogenem Aussehen, die sich nach van Gieson gleich wie in unsern Präparaten heller als die Lamellen der Cornea färbt und nur, wo sie bogenförmig von der Bow man schen Membran losgedrängt durch die Epithelzellen nach oben zieht, eine fibrilläre Struktur zeigt.

Die anatomische Untersuchung dieses Falles von Keratitis bullosa berechtigt wohl zu der Forderung, dass die ganze Cornea in fortlaufenden Schnitten aufs sorgfältigste zu untersuchen ist, ehe eine in grösserer Ausdehnung vorhandene subepitheliale homogene Gewebs- 
schicht als eine Lage geronnener Flüssigkeit angesprochen werden darf. Hiermit soll keineswegs das Vorkommen einer solchen bei Keratitis bullosa schlechtweg geleugnet werden.

Das meiste Interesse gewinnen wir vorliegendem Fall aber durch einen Vergleich des klinischen und anatomischen Bildes ab. Wissen wir doch, wie noch Foster (25) hervorgehoben hat, über das klinische Bild des Pannus degenerativus so gut wie nichts, da es sich bisher stets um zufällige anatomische Befunde gehandelt hat. Nur Fuchs (3) erwähnt einmal, dass ,in sehr alten Fällen von Glaukom endlich solide Auflagerungen einer durchscheinenden glasigen Substanz auf der Oberfläche der Hornhaut entstehen, gerade als ob es schliesslich zur Konsolidierung der ursprünglich bläschenartigen Gebilde käme. Auch pflegt dann Gefässentwicklung vom Rande der Hornhaut in diese aufgelagerten Massen hinein stattzufinden".

Dieser Schilderung scheinen klinische Beobachtungen zugrunde zu liegen; ob gerade solche Fälle zur anatomischen Untersuchung kamen, geht aus den weiteren Ausführungen mit Sicherheit nicht hervor. Jedenfalls dürfte gerade in solchen alten Fällen von Glaukom, bei denen schon häufige Schmerzanfälle und wiederholte Blaseneruptionen stattgefunden haben, ein pannösèr Überzug erwartet werden, auch wenn Gefässbildung klinisch nicht nachgewiesen werden kann.

Meller(49) fand sodann als Grundlage einer weisslichen, anscheinend gefässlosen Hornhauttrübung eine derbe subepitheliale Bindegewebsschicht, deren sichere Lokalisation kliniseh nicht möglich gewesen war.

Das matte gestippte Aussehen der Cornealoberfläche bei unserem Fall ist nicht durch den Pannus bedingt, vielmehr die Folge von Epithelveränderungen, wie sie der Keratitis bullosa eigentümlich sind (siehe unten).

Die einzige klinisch wahrnehmbare Veränderung, die auf den Pannus zurückgefïhrt werden kann, ist die grauweissliche Verfärbung des temporalen Limbus (vgl. Meller loc. cit.), und diese war wegen des Fehlens von Gefässen klinisch nicht für pannös gehalten, sondern als Infiltration der vorderen Corneallamellen gedeutet worden. Es kann somit ein degenerativer Pannus wohl ausgebild et vorhanden sein, ohne dass er klinisch deutlich in Erscheinung tritt, und zwar wird es sich dann um solche seit längeren Jahrenerblindete Augen handeln, wo das Fehlen der Gefässe und die Zellarmut des Pannus es wohl erklärlich scheinen lassen, dass dieTransparenz der Cornea nirgends beeinträchtigt war. In dieser auf- 
fallenden Geringfügigkeit der klinischen Merkmale wird man vielleicht auch einen Unterschied des älteren degenerativen Pannus vom älteren skrofulösen und pannösen Pannus erblicken dürfen. Denn wenn diese beiden auch einer ausserordentlich weitgehenden Rückbildung fähig sind, so wird man doch mit dem Hornhantmikroskop fast stets noch feine Gefässchen und zarte oberflächliche Trübungen finden, die der bei skrofulöser Keratitis stets vom Parenchym ausgehende, bei pannöser trachomatöser Keratitis ebenfalls häufig im Parenchym sich abspielende Prozess hinterlassen hat. Natürlich gilt das Gesagte nur für den älteren narbigen Pannus degenerativus, der sklerotisch, zellarm und homogen geworden ist. Das klinische Bild wird ein ganz anderes sein, wenn die Veränderungen jüngeren Datums sind. Gefässentwicklung und Zellreichtum werden dann zu einer mehr oder weniger erheblichen Beeinträchtigung der Transparenz der Cornea führen.

Nachdem Baas (12) das häufige Vorkommen dieses Pannus an degenerierten Augen auf Grund anatomischer Untersuchungen hervorgehoben hat, wird man nunmehr in entsprechenden Fällen auch die klinische Diagnose stellen dürfen. So hatte ich Gelegenheit, am rechten Auge eines infolge Blennorrhoea neonatorum beiderseits er-blindeten Mädchens eine Veränderung zu beobachten, die ich mit Sicherheit für bedingt durch einen Pannus degenerativus halten möchte.

Emma L., 8 Jahre alt, zeigt beiderseits zentrale Hornhautlenkome mit Irisadbärenz. Rechts ist die Cornea vergrössert (vertikal 13, horizontal $12 \mathrm{~mm}$ ) und ektatisch. In der Mitte der Cornea finden sich hyaline Einlagerungen. Das Epithel der unteren Hornhanthälfte vom Limbus bis dicht unterhalb dieser Einlagerungen ist schwielig verdickt; unter dem Epithel ziehen zahlreiche Gefässe bis zur Hornhautmitte. Epithelverdickung und Vaskularisation schneiden dicht unterhalb der Einlagerungen scharf $a b$ und prominieren dentlich gegenüber der tiefer liegenden Fortsetzung des Epithels nach oben zu. Diese subepitheliale Schicht stellt ein grauliches, gefässhaltiges, wenig durchsichtiges Gewebe dar und liegt jedenfalls über dem in der Peripherie noch erhaltenen Cornealgrundgewebe. Nach seiner oberflächlichen Lage und seiner Genese ist dies Gewebe als Pannus degenerativus zu bezeichnen.

Das hier beobachtete Bild ähnelt wegen der schwieligen Epithelverdickung mehr dem trachomatösen als dem skrofulösen Pannus; ersterem steht der degenerative Pannus ja auch anatomisch näher.

Die anatomische Untersuchung unseres Falles gibt aber noch weitere Aufschlüsse zur Klinik des Pannus degenerativus. Ich meine die Tatsache, dass dieser Pannus an einem Auge sich findet, welches klinisch das Bild der Keratitis bullosa geboten hat, und zwar scheint 
ein gewisses Verhältnis zwischen Blasenbildung und Pannus zu bestehen derart, dass der eine die andere ausschliesst: wo die pannöse, subepitheliale Schicht kräftig entwickelt ist, finden sich im Epithel keine Zerfallserscheinungen, und umgekehrt, wo Epitheldegeneration vorliegt, ist die pannöse Lage sehr schwach entwickelt, ja fehlt meist ganz.

Die Entwicklung einer subepithelialen bindegewebigen Schicht ist nun schon mehrfach bei Keratitis bullosa beobachtet worden, ohne dass dieser Koinzidenz der anatomischen Bilder bisher eine erhebliche Bedeutung beigemessen worden wäre. Im folgenden seien daher die diesbezüglichen Mitteilungen kurz zusammengestellt.

Während Leber (1) mitunter bei Augen mit glaukomatösen Zuständen zwischen Epithel und Bowmanscher Membran eine dünne neugebildete Bindegewebsschicht fand, welche sich dadurch vom Pannus unterschied, dass Gefässe oft vermisst wurden, erwähnt Fuchs (3) als Erster ausdrücklich, dass man bei Keratitis bullosa über der Bowmanschen Membran „eine Bindegewebsschicht findet, gebildet aus parallelen und ziemlich homogenen Fasern, welche mit dem eigentlichen Hornhautgewebe grosse Ähnlichkeit haben. Diese Schicht ist von der Bow manschen Membran durch eine Flüssigkeitsansammlung getrennt und stellt die vordere Wand der Blase dar". Vom Rande der Hornhaut ziehen feinste Gefässe gegen die Stelle der Blase hin, deren jedes von einer Hülle lockeren Bindegewebes umgeben ist. Zur gleichen Zeit erwähnt Arlt(28), dass bei Keratitis bullosa die Blasenwand nicht aus der Bow manschen Membran und der Epithelschicht oder aus letzterer allein bestehe, sondern aus einer zwischen beide eingeschalteten neugebildeten Gewebsschicht. Sodann erwähnt Tartuferi(29), dass die Blasenwand bei Keratitis bullosa auch aus einem subepithelialen neugebildeten Bindegewebe vor der Bowmanschen Membran bestehen kann. Allerdings ist aus dem Referat in Nagels Jahresbericht nicht zu ersehen, ob es sich um eine vom Limbus herkommende Schicht handelte. Dagegen spricht Bock(30) ausdrücklich davon, dass die Rundzellen, um subepithelial an der Hornhautoberfläche zu erscheinen, am häufigsten den Weg von der Peripherie her nehmen. ( ${ }_{y}$, Wucherung im Conjunctivalblatte der Cornea von der Bindehaut des Bulbus aus mit darauffolgender Bildung einer pannösen Schicht.") Die von Brugger(31) unter einer grossen Blase beobachtete subepitheliale Gewebsmasse von stellenweise lamellösem Bau scheint wiederum lokal entstanden zu sein, hierfür spricht auch das Fehlen der Bowmanschen Membran unter dieser Gewebsmasse. 
Bossalino(32) hat sodann zwischen der Bowmanschen Membran und dem Epithel in einem neugebildeten pannösen Gewebe Gefässe gesehen, "welche sich sehr zahlreich, besonders in der Peripherie der Cornea, zeigen, angefüllt mit roten Blutkörperchén und umgeben von einer ziemlich grossen Lymphzelleninfiltration". Auch v. Hippel(50) erwähnt ein pannöses Gewebe von geringer Mächtigkeit vor der Bow manschen Membran bei Keratitis bullosa. Ewing(33), der vier Stadien der Keratitis bullosa annimmt, auf die ich erst später einzugehen haben werde, beschreibt im letzten Stadium als Inhalt der Blase Fibrin, Wanderzellen, Spindelzellen; schliesslich wachsen auch noch Blutgefässe in die Blase hinein. De Schweinitz und Shumway (27) fanden bei Keratitis bullosa an einem glaukomatösen Auge am Limbus das Epithel von der Bowmanschen Membran durch viele Rundzellen getrennt, in welche gefässhaltiges, pannöses Bindegewebe eingebettet ist. Auch Meller(49) hebt hervor, dass der Pannus, den er an einem Auge mit Jahre lang rezidivierender Keratitis bullosa beobachtete, zweifellos vom Limbus ausgegangen sei. Bemerkenswert erscheint, dass auch Meller Epithelveränderungen nur dort sah, wo sich statt der dicken zellarmen Bindegewebsschicht eine dünne Lage kleinzelligen Gewebes mit Blutgefässen fand.

Etwas ausführlicher behandelt dieses pannöse Gewebe aber allein Wirths, der drei Fälle von Keratitis bullosa untersuchte, allerdings nur den dritten an Serienschnitten. In diesem dritten Falle ziehen ,aus dem pannösen Gewebe am Limbus entspringend, meist längs-, aber auch quer getroffene Gefässe zwischen Epithel und Bowmansche Membran, daneben finden sich Bindegewebsfasern mit spindelförmigen, den Hornhautkörperchen ähnlichen Zellen, sowie Lymphound polynucleäre Leukocyten. Die Anzahl der Zellen nimmt mit der Entfernung vom Limbus allmählich ab. Beim Beginn der Blase verbreitert sich dieses Gewebe und reicht in dem Schnitte, der die grösste Ausdehnung der Blase trifft, bis zum andern verdickten Ende derselben, um welches es sich herum zu schlingen scheint. Es besteht hier aus wenig lichtbrechenden, zum Teil gequollen aussehenden Faserbündeln, zwischen denen sich zahlreiche Hohlräume und Spalten finden, und ist bedeutend schwächer gefärbt wie das Hornhautparenchym. Die Zellen liegen unregelmässig verteilt; sie sind meist spindelförmig und mit einem länglichen Kern versehen, doch finden sich auch sternförmig verästelte sowie mehr rundliche Zellformen. Das ganze Gewebe ist nur wenig von der Bowmanschen Membran abgehoben".

Sehen wir nun auch von den Fällen Tartuferis, Bruggers, v. Graefe's Arehiv fin Ophthalmologie. LXIX. I. 
v. Hippels und Ewings ab, bei denen die Herkunft der neugebildeten Bindegewebsschicht vom Limbus mir nicht sicher erwiesen scheint oder nicht ausdrücklich erwähnt wird, so bleiben immer die Beobachtungen von Fuchs, Bock, Bossalino, de Schweinitz und Shumway, Meller, Wirths und wahrscheinlich auch Arlt verwertbar, die bei Keratitis bullosa ein subepitheliales, pannöses, vom Limbus her entwickeltes Gewebe antrafen. Aber nur Wirths widmet der Deutung dieses Befundes einige Worte und zwar nimmt er an, dass dieser degenerative Pannus „im äussersten $\mathrm{Ab}$ schnitt der Blase bei Bildung derselben bereits vorhanden war und von hier aus dann in ihre übrigen Teile hineingewachsen ist". Die Einsprossung des pannösen Gewebes in den Blasenhohlraum würde also nach dieser Auffassung zur Organisierung und Konsolidierung der Hohlräume führen. Diese Bemerkung von Wirths erscheint mir um so wichtiger, als ähnliche Beziehungen in dem Verhältnis zwischen Blasenbildung und Pannus auch mir unverkennbar scheinen; denu, wie schon oben hervorgehoben, findet sich die pannöse Schicht fast ausschliesslich nur dort, wo kein Epithelzerfall beobachtet wird. Da die rauchige Hornhauttrübung und die stets wiederkehrenden Schmerzanfälle bei unserem Fall schon sechs Jahre vor der Enucleation beqbachtet worden waren, da wir ferner vielfach neugebildetes Epithel vor uns haben, möchte ich annehmen, dass die Epithelveränderungen ursprünglich wie in andern Fällen von Keratitis bullosa über ein grösseres Hornhautareal ausgedehnt waren. Mit der nun allmählich immer weiter um sich greifenden Gewebsausbreitung, vom Limbus her wurde der Durchtritt der Ödemflüssigkeit - welcher jedenfalls einen Faktor für die Blasenbildung abgibt - erschwert und die schliesslich bleibende Epithelbedeckung über der pannösen Lage ist dauerhafter und solider, frei von bullöser Degeneration. Der beste Beweis für die Richtigkeit dieses Schlusses sind die Zerfallerscheinungen im Epithel desselben Auges, dort, wo der Pannus fehlt.

An und für sich brauchte das gemeinsame Vorkommen von Pannus degenerativus und bullöser Epithelentartung ja nicht in innere Beziehung gebracht zu werden; handelt es sich doch bei beiden um anatomische Bilder, deren jedes isoliert und primär an degenerierten Augen beobachtet wird. Die Art der Kombination beider Veränderungen in unserem Falle, wo der Pannus bis in die Nähe des Epithelzerfalls sich vorschiebt, deutet aber darauf hin, dass die Blasenbildung der primäre, Pannusentwicklung der sekundäre Vorgang ist.

Tritt somit bei Keratitis bullosa anatomisch nachweis- 
bar Pannusbildung auf, so wird man hierin einen anatomischen Heilungsvorgang erblicken dürfen. Dass beide Veränderungen - Pannus und Epithelzerfall - nebeneinander gefunden werden und nicht etwa das Stadium der Pannusbildung allein, erklärt sich zur Genïge dadurch, dass die Schmerzhaftigkeit der Blasenbildung und anderer degenerativer Prozesse im Auge zur Enucleation fübren wird, bevor-noch die anatomische Heilung vollendet und damit die Keratitis bullosa abgelaufen ist.

Um den Beziehungen zwischen Keratitis bullosa und Pannusbildung weiter nachzugehen und um ferner die in der Literatur vorhandenen Widersprüche bezüglich des anatomischen Substrats der Keratitis bullosa an der Hand eines grösseren Materials zu prüfen, untersuchte ich weitere fünf Augen, die klinisch entweder grössere oder auch kleinere Bläschen und Unebenheiten der Cornealoberfläche (Keratitis vesiculosa) gezeigt hatten. Wenn nicht jedesmal ausdrücklich von Keratitis bullosa und vesiculosa die Rede ist, so ist das damit zu begründen, dass letztere häufig als Vorläufer und auch neben der Keratitis bullosa am gleichen Auge vorkommt, mit dieser also wesensgleich ist, wie auch Fuchs(3) (S. 70) erwähnt. Augen mit Cornealbläschen bei Katarrh der Bindehaut, der Respirationsorgane und bei Herpes zoster (Keratitis vesiculosa nach Saemisch) standen mir nicht zur Verfügung.

\section{Fall II.}

Die 52 Jahre alte Patientin war bei der I. Aufnahme in die Privataugenheilanstalt des Herrn Geheimrat Saemisch im Januar 1903 schon seit einiger Zeit auf dem rechten Auge erblindet. Das Auge bietet den Status glaucomatosus dar: ausgesprochene Keratitis bullosa, besonders über den mittleren Teilen der Cornea, maximal weite Pupille, hochgradige Sehnervenexcavation, später auch Katarakt. Enucleation am 3. V. 1903. Fixation in Müllerseher Flüssigkeit. Der Bulbus wird vertikal aufgeschnitten, die nasale Hälfte in Celloidin eingebettet und in $20 \mu$ dicke Serienschnitte zerlegt; die temporale Cornealhälfte wird in Paraffin eingebettet und in Serien von $10 \mu$ geschnitten.

\section{Mikroskopischer Befund.}

Aus dem Befund, soweit er nicht die Cornea betrifft, sei nur mitgeteilt, dass typische glaukomatöse Sehnervenexcavation von $1,5 \mathrm{~mm}$ Tiefe ohne nachweisbare Höhlenbildung vorliegt. Die Iris ist in maximaler Mydriasis auf einen kurzen, dicken, sehr zellreichen Stumpf retrabiert. Die Kammerbucht ist unten aufgehoben, da sich zwischen Iriswurzel und Descemetsche Membran eine mächtige Rundzellenansammlung gelagert hat, in der sich auch vereinzelte Riesenzellen vorfinden. Oben besteht operatives 
Iriscolobom. Die Linse zeigt subkapsulär und supranucleär kataraktösen Zerfall.

Die Cornea weist nur oben in der Nähe der Schnittnarbe zellige Infiltration des Parenchyms auf; ebendort ist die Descemetsche Membran unterbrochen, die Cornea stark verdünnt und nach aussen leicht vorgewölbt. Die Schnittränder sind innen nicht glatt vereinigt, sondern klaffen infolge des vermehrt gewesenen intraokularen Druckes und sind nur teilweise durch eine schwach sich färbende zellarme Bindesubstanz verbunden. Die Corneallamellen sind vielfach in den tieferen und mittleren Lagen des Parenchyms auseinander gedrängt; nur selten findet man aber in den so entstandenen Lücken geronnene Flüssigkeit. Näher der Oberfläche unter der Bowmanschen Membran sind solche Spalträume nicht vorhanden. Unweit vom oberen Hornhautrande findet man unter dem Conjunctivalepithel reichlich Rundzellenansammlungen, welche bis zum Limbus reichen; spärlicher eingestreat sieht man auch in der Nähe des unteren Limbus Rundzellen. Von hier und zwar hauptsächlich in der nasalen Bulbushälfte setzt sich zwischen Bow man sche Membran und Hornhautepithel eine sehr schmale Gewebsschicht anf die Cornea fort, die sich alsbald erheblich, auf die zwei- bis dreifache Dicke der Bowmanschen Membran verstärkt, fibrillären Bau erkennen lässt and ziemlich reich an fixen Bindegewebszellen und eingestrenten Leukocyten ist. In der unteren Randzone der Cornea sieht man diese subepitheliale Gewebsschicht bis zu $3-4 \mathrm{~mm}$ vom Limbus aus anf die Cornea sich erstrecken; Gefässe sind in ihr nicht nachweisbar. Auch am oberen Hornhautrande findet sich eine solche subepitheliale Lage; sie trägt hier zumeist den Charakter des an Rundzellen reicheren, faserarmen Granulationsgewebes; jedoch auch hier sind keine Gefässe vorhanden. Der Pannus setzt sich hier nur 1-2 mm über den Hornhautrand fort. In der Năhe des horizontalen Meridians fehlt diese pannöse Schicht in der nasalen Bulbushälfte so gut wie völlig und hier begegnet man weitgehenden Epithelveranderungen. In der temporalen Bulbushälfte ist die subepitheliale Schicht am Limbus überhaupt viel sehwächer ausgebildet und reicht nicht soweit wie nasal; dementsprechend geht der Epithelzerfall hier weiter bis zur Peripherie.

Ubergehend auf die Veränderungen im Epithel selbst wäre zunächst zu erwähnen, dass in der mittleren Schicht des Conjunctivalepithels am unteren Limbus sich grosse, aus mehreren Zellen hervorgegangene Hohlräume finden, in denen. sich als einzige Überreste des Inhalts einzelne Protoplasmafäden zejgen.

Das Hornhautepithel zeigt in den dem Limbus benachbarten Partien relativ die geringsten Veränderungen. Es besteht hier aus einer basalen Schicht hoher cylindrischer Zellen, zwischen denen sich vielfach ungefärbte Zwischenräume finden. Diese stellep aber nur teilweise erweiterte Intercellularräume dar, ein anderer Teil ist so entstanden, dass das Protoplasma konzentrisch zum Kern schrumpfte und so zwischen den Zellen ungefärbte $Z$ wischenräume entstehen liess. Hier und da fehlt die Schicht der Stachel- und Riffzellen völlig; es folgen danu unmittelbar auf die Basalzellenlage die flachen oberfächlichen Epithelreihen. 
Uber den mittleren Teilen der Cornea finden sich nun reichlich degenerative Veränderungen in der Epithelschicht, welche in dieser Region in der nasalen Bulbushälfte der Bow manschen Membran unmittelbar aufliegt, während in der temporalen Hälfte auch hier stellenweise eine subepitheliale Gewebsschicht beobachtet wird. An Stelle der cylindrischen Basalzellen trifft man kleinere und grössere Hohlräume, welche aus ein, zwei und mehr Basalzellen hervorgegangen sind, deren Protoplasma bis auf verschwindende Reste zugrunde gegangen ist (Taf. I, Fig. 6). Diese Reste haben sich teils um den Kern zusammengezogen, teils durchziehen sie als feine Protoplasmafäden den ganzen Hohlraum, die ursprünglichen Zellgrenzen noch andeutend. Der Kern liegt vielfach fast frei inmitten des Hohlraumes; ein Teil der Kerne ist aber auch völlig zugrunde gegangen, so dass ein Teil der blasigen Hohlräume gar keinen Kern enthält, während andere aus mehreren Zellen hervorgegangene Hohlräume nur ein bis zwei Kerne enthalten (Taf. I, Fig. 6). Sonst ist ein Inbalt in diesen Hohlräumen nicht wahrzunehmen. Die äusseren Epithellagen sind stellenweise normal, an andern Stellen ist die ganze Epithelschicht in Blasenbildung aufgegangen. Grosse Hohlräume liegen dann oberflächlich nur von einem schmalen Protoplasmasaum bedeckt (Taf. I, Fig. 7); wieder an andern Stellen ist die Blase gesprungen und somit frei gegen die Oberfläche geöffnet. In der temporalen Bulbushälfte zieht mehrfach die auf zwei Zellreihen reduzierte Epithelsehicht bogenförmig über völlig leere HohIränme, die zwischen ihr and der Bowmanschen Membran gelegen sind, hinweg.

An der Grenze vom mittleren zum - unteren Cornealdrittel sind diese Veränderungen am lebhaftesten ausgeprägt und zwar hauptsächlich temporal, und hier findet sich eine sehr zarte dünne subepitheliale Gewebslage von teils locker maschigem, teils auch fibrillärem Bau, die stellenweise isoliert scheint, an andern Stellen aber in deutlichem Zusammenhang mit dem vom unteren Limbus her kommenden Gewebe steht.

Am Übergang vom unteren zum mittleren Cornealdrittel hört die Hohlraumbildung im Epithel fast völlig auf, und nach der Cornealmitte zu finden wir ein neugebildetes, aus unregelmässigen polygonalen Zellen bestehendes Epithel von ein bis zwei, stellenweise anch mehr Lagen. Dies Epithel ist von der Bowmanschen Membran durch eine recht lockere und zellarme Gewebslage getrennt (Taf. I, Fig. 7), welche sich mit Eosin and nach van Gieson wie das Cornealparenchym, schwächer als die Bowmansche Membran färbt und bis $1 / 8$ der Dicke des Cornealparenchyms erreicht. Es handelt sich um ein sehr.lockeres schwammiges Gewebe, zwischen dessen vielfach sich durchtlechtenden Fibrillen sich auch freie ungefärbte Hohlräume finden, während an andern Stellen dies Gewebe noch einige degenerierte Epithelien umwächst. Die lockere maschige Bindegewebslage greift auch noch in die Randzone der Epitheldegeneration hinein; hier schiebt sie sich zwischen die Hohiräume und sprosst, sie auskleidend, in ihr Inneres hinein (Taf. I, Fig. 7). Auch die Epitheldecke sendet hier und da Sprossen und Ausläufer in die Tiefe zwischen die Maschen des Gewebes. Diese subepitheliale Schicht färbt sich nach Mallory-Ribbert and Fränkel wie Bindegewebe. 
Auf kurze Strecke in der Nähe des horizontalen Meridians liegt die vordere Grenzlamelle auch ganz frei zutage, indem die zuvor degenerierten und abgestossenen Epithelien von der Nachbarschaft her noch nicht durch neue ergänzt worden sind, und auch die pannöse Schicht noch nicht bis dahin vorgedrungen ist. In der Nähe dieses Defektes sind die von der Seite herüberragenden Epithelien schlecht gefärbt, gequollen, zum Teil auch in stark lichtbrechende blasige Gebilde umgewandelt (wie an der analogen Stelle bei Fall I, S. 6).

Unweit von diesem Epitheldefekt sieht man auf etwa 10 Schnitten $(100 \mu)$ ein zartes kernfreies intraepitheliales Gewebsband, welches wie bei Fall I von der subepithelialen Schicht abzweigt, über eine kleine Gruppe von der Seite darunter gewucherter Epithelien und unter den basalen Cylinderzellen verläuft und sich alsbald wieder der Bowmanschen Membran auflegt (vgl. die Fig. 3 u. 5 auf Taf. I von Fall I). Dies Band besitzt etwa $1 / 5-1 / 1$ der Dicke der vorderen Grenzlamelle.

Die Bowmansche Membran ist überall ganz intakt; nur ganz vereinzelt sieht man sie von erweiterten Nervenkanälchen durchsetzt.

\section{Fall III.}

Die Untersuchung dieses Auges eines 80jährigen Mannes mit Glankom und Keratitis bullosa ergibt einen Befund, der dem der beiden vorher mitgeteilten Fälle vollkommen entspricht. Deshalb kann von einer ausführlichen Mitteilung Abstand genommen werden. Die Epithelveränderungen treten hier wieder wie im ersten Falle gegenüber der Pannusentwicklung zurück; wo sie vorhanden sind, fehlt der Pannus, und wo man dem ziemlich sklerotischen Pannus begegnet, da findet sich auch eine solide Epitheldecke. Die Transparenz der Cornea war durch die Pannusentwicklung nicht beeinträchtigt worden.

Bevor wir zu den Befunden der weiteren Fälle von Keratitis bullosa uibergehen, bei denen eine pannöse Schicht nicht zux Beobachtung kam, sei nur mit. wenigen Worten auf die Beziehungen hingewiesen, die Fall II und III mit dem an erster Stelle ausführlich behandelten gemein haben. Bei Fall I und III finden wir als hauptsächlichste Veränderung den Pannus, neben ihm spielt der Epithelzerfall nur eine geringe Rolle; für Fall II liegt das Verbältnis umgekehrt: in den mittleren Teilen der Hornhaut findet sich fast ausschliesslich Epithelzerfall und Hohlraumbildung, wäbrend die pannöse Schicht im wesentlichen auf die Randteile der Hornhant beschränkt bleibt; dabei trägt die subepitheliale Lage hier noch stellenweise den Charakter des Granulationsgewebes, während strafferes Bindegewebe in viel geringerem Masse, homogene kernfreie Partien überhaupt nicht vorkommen. Es handelt sich somit bei Fall II um ein jüngeres Stadium des degenerativen Pannus, sowohl nach seinem 
Bau wie nach seiner vornehmlich auf die Randzone beschränkten Lage. Hiermit steht im besten Einklang, dass das Epithel in viel grösserem Umfange Zerfallserscheinungen zeigt als bei Fall I und III. In der Epithelzerfallszone fehlt die Bindegewebsschicht fast ausnahmslos, nur an der Grenze des unteren und mittleren Cornealdrittels greift sie in die epithelialen Hohlräume hinein. Auf diese Weise kommt es also zu einer Organisierung des blasigen Hohlraums, wie sie auch Wirths beobachtet hat.

Dass diese Einwucherung der pannösen Schicht in die epithelialen Zerfallshöhlen des Falles I sich nur an der Übergangsstelle zu der solideren Epitheldecke vollzieht, entspricht unserer schon beim ersten Fall gewonnenen und durch den III. Fall bestätigten Anschauung, wonach der Pannusbildung eine Heilungstendenz beizumessen ist, indem sie wieder die Neubildung einer soliden, widerstandsfähigen Epitheldecke zur Folge hat.

Aber nicht nur der sekundär entwickelte Pannus bei Keratitis bullosa stellt einen anatomischen Heilungsvorgang dar. Bei der Untersuchung mehrerer Augen mit grossem Totalstaphylom der Cornea begegnete mir an einem riesigen Auge ein stark entwickelter Pannus, der den grössten Teil der vorderen Bulbuswand bildete. Dieser Fall von Staphylom mag hier wegen seiner Beziehung zum Pannus degenerativus eingeschaltet werden, zumal er sowohl klinisch wie anatomisch von dem für Totalstaphylome der Hornhaut bekannten Bilde abweicht.

Clara $H$. erblindete als Kind auf dem linken Auge infolge von Hornhautgeschwüren. Im Jahre 1888 war das rechte Auge normal und im Besitze voller Sehschärfe. 1890 soll das rechte Auge im Verlauf eines Typhus unter heftigen Schmerzen erkrankt sein; anfangs der neunziger Jahre erblindete auch dies Auge völlig.

1905 Aufnahme des nun 29 Jahre alten Mädehens. Die rechte Hornhaut stellt eine fast ganz regelmässig vorgewölbte Halbkugel dar, die leicht grau getrübt und von Gefässen oberfläcllich durchsetzt ist; im allgemeinen ist sie aber noch ziemlich gut transparent. Der Limbus ist sehr stark verbreitert. Von Limbus zu Limbus beträgt der Durchmesser $25 \mathrm{~mm}$. Oben ist eine riesige Vorderkammer erbalten, unten ist die Iris mit der Hornhaut verwachsen. Die Pupille hat einen Durchmesser von nahezu $8 \mathrm{~mm}$. Die Mitte des Pupillargebietes wird von einer verkalkten Linsenmasse eingenommen, die bis zur Hornhauthinterfläche reicht.

Das enucleierte Auge hat eine Achsenlänge von $40 \mathrm{~mm}$. Die temporale Cornealhälfte wird in Paraffin eingebettet, die nasale der Celloidintrockenmethode unterworfen. 


\section{Mikroskopischer Befund.}

Die Cornea ist stark verdünnt, in den mittleren Teilen bis auf etwa $0,25 \mathrm{~mm}$, also nahezu $1 / 4$ der normalen Dicke. Der Hauptteil der Membran besteht aus einem ziemlich zell- und gefässreichen Pannus mit wenig Kalkeinlagerung. Er liegt zwischen dem Epithel und der grösstenteils gat erhaltenen Bowmanschen Membran und hat eine überall fast gleiche Stärke von etwa $0,2 \mathrm{~mm}$. In den mittleren Teilen der Cornea, wo das Parenchym nur aus wenigen Lamellen besteht, macht der Pannus also nahezu ${ }^{4} / 5$ der Dicke der Cornea aus. Die D escemetsche Membran ist in der Peripherie nicht überall erkennbar, sonst aber auf grosse Ausdehnung hin erhalten, nur zentral fehlt sie wieder anf eine Strecke von etwa 2 qmm; hier ist die Spitze einer Pyramidalkatarakt mit der Hornhauthinterfäche fest verwachsen; auf ihren Rand schlagen sich die Enden der Descemetschen Membran um; an Stelle der Hornhautlamellen findet sich hier ein ziemlich zellreiches Narbengewebe bei ebenfalls fehlender Bowmanscher Membran; das Epithel treibt hier besonders zahlreiche Zapfen in die Tiefe.

Die Iris ist unten in ziemlicher Ausdehnung mit der Hornhaut verwachsen, aber fast uberall in ihrer Struktur noch erkennbar, nur ganz in der Peripherie ist sie bis auf die Pigmentlage reduziert; oben besteht nur im Bereich des Kammerwinkels periphere Verwachsung. Im übrigen ist die Vorderkammer sehr tief, in der Mitte neben der Pyramidalkatarakt bis $\mathrm{zu} 4 \mathrm{~mm}$.

Dieses "Totalstaphylom" der Cornea erregte schon klinisch ein gewisses Interesse, weil die Vorderkammer zum Teil erhalten, die Pupille sogar ganz frei von Verwachsung mit der Cornea geblieben war; dieser Befund wurde durch die anatomische Untersuchung erhärtet. Trotzdem handelt es sich nicht um eine Keratektasia, eine Vorwölbung der Cornea ohne Perforation, wie man wegen der Erhaltung der Vorderkammer und wegen der verhältnismässig guten Transparenz annehmen könnte, denn die anatomische Untersuchung erbrachte den Beweis, dass eine zentrale Perforation bestanden hat, in deren Folge es zu Pyramidalkatarakt gekommen ist. Bisher findet sich übereinstimmend angegeben ${ }^{1}$ ), dass die Wandung des Staphyloms von einem Narbengewebe gebildet werde, welches aus der prolabierten Iris hervorgegangen ist. Hornhautgewebe findet sich höchstens noch spärlich in den Randpartien des Staphyloms²).

1) Siehe Saemisch loc. cit. S. 290, Greeff loc. cit. S. 191.

2) Anmerk. bei der Korrektur. Vgl. die Ausführungen E. v. Hippels über die Entstehungsweise angeborener Staphylome und Leukome. Dieses Archiv. Bd. LXVIII. S. 378. Da in unserm Falle die Trübung dex Hornhant zart, keineswegs leukomartig war, durfte die Diagnose anch nicht auf ektatisches Leukom mit Erhaltung der Vorderkammer gestellt werden. Vielmehr besteht sowohl klinisch wie anatomisch noch die grösste Ähnlichkeit mit dem Staphylom. 
Das ungewöhnliche Bild unseres Falles ist wohl dadurch zu stande gekommen, dass sich die Pyramidalkatarakt gebildet hat; so blieb die Vorderkammer teilweise erhalten. Erhalten blieben ferner mit Ausnahme der zentralen Perforationsstelle das enorm gedehnte und verdïnnte Cornealparenchym. Den grössten Teil der vorderen Bulbuswandung macht aber die gleichmässig über die ganze Cornea zwischen Epithel und Bowmanscher Membran entwickelte Gewebsschicht aus, die einen Pannus degenerativus darstellt.

Die Entwicklung dieses Pannus darf wohl als ein Reparationsvorgang aufgefasst werden. Denn die enorm verdünnte Cornea würde wohl kaum den Insulten des Lidschlages bei gesteigertem Binnendruck standgehalten haben. So kam es gleichzeitig mit zunehmender Ektasie zu reparativer Verstärkung der gedehnten vorderen Bulbuswand durch Entwicklung eines subepithelialen Pannus. Demselben kann für dieses staphylomatöse Auge ebenso wie bei den Augen mit Keratitis bullosa die Bedeutung eines heilenden Faktors beigelegt werden.

\section{Fall IV.}

Das Auge stammt von einer äIteren Frau, die an chronischer Iridochorioiditis, Sekundärglaukom und Keratitis bullosa gelitten hatte.

Der in Formalin fixierte und in Alkohol nachgehärtete Bulbus ist vertikal aufgeschnitten. Von der einen schon früher in Celloidin eingebetteten Bulbushälfte stellte mir mein Freund, Privatdozent Dr. Reis 30 Schnitte freundlichst zarVerfügung, die leicht der Reihe nach geordnet werden konnten, da Pupille und Papille in den Schnitten getroffen, schnelle Orientierung ermöglichten. Von der andern Bulbushälfte, welche etwas mehr als die Hälfte der Cornea enthielt, wurde die Cornea mit Iris und Proc. ciliares abgetrennt und in Paraffin eingebettet. Lückenlose Schnittserie von 6 , später $10 \mu$.

Mikroskopischer Befund.

Aus dem allgemeinen Befunde sei nur hervorgehoben, dass die Iriswurzel der Hornhauthinterfläche adhäriert, so dass die Kammerbucht aufgehoben ist. Der Pupillarrand der atrophischen Iris ist unten mit der vorderen Linsenkapsel verwachsen. Die inneren Netzhautschichten sind in der Gegend des hinteren Pols stellenweise bindegewebig entartet. Es besteht nur geringgradige Excavatio glaucomatosa der Pap. nervi optici. In der Duralscheide des N. opticus finden sich zahlreiche geschichtete Konkremente.

Das Cornealparenchym zeigt in den hinteren und mittleren Lagen etwas Auflockerung der Lamellen, ist aber frei von entzündlichen Veränderungen.

Die Bowmansche Membran ist überall intakt; zwischen ihr und dem Epithel findet sich nirgends eine eingewucherte Gewebsschicht.

Das Hornhautepithel zeigt über die ganze Membran ausgebreitet ausserordentlich hochgradige Veränderungen, so dass nirgends eine normale 
fünfreihige Epitheldecke zu sehen ist. An Stelle der Basalzellènlage sieht man überall mit alleiniger Ausnahme der Randteile der Cornea mehr oder weniger grosse cystenartige Hohlränme unmittelbar über der Bowmanschen Membran. Diese Hohlräume sind entweder völlig inhaltfrei und leer oder zeigen besonders an ihren Seiten noch feine Protoplasmafäden, die sie spinnewebartig durchziehen (vgl. die Fig. 6 auf Taf. I, die die allerdings weniger hochgradigen Veränderungen des Falles II wiedergibt). Zuweilen finden sich noch lebhaft tingierte Zellkerne in diesen Hohlräumen; nur vereinzelt hingegen sieht man in ihnen eine sehr schwach mit Eosin sich färbende homogene Masse. Meist zieht über diese Hohlräume eine ein-, selten auch zweireihige Lage von platten oberflächlichen Zellen; die Stachel- und Riffzellen fehlen also völlig. Vielfach ist aber auch die gesamte Epitheldecke in die Hohlraumbildung aufgegangen, so dass sich als vordere Begrenzung dieser cystenartigen Räume nur eine schmale protoplasmatische Membran findet.

In den Randteilen der Hornhant ist diese Hohlraumbildung in Epithel noch nicht so ausgeprägt, man erkennt vielmehr noch häufig die cylindrischen Basalzellen, die aber erheblich verändert sind. 'Sie zeigen nämlich einen langen schmalen, schwachgefärbten Protoplasmaleib, an dessen vorderem, von der Bowmanschen Membran abgewandten Ende der Kern sitzt [Keulenzellen Lotts (47)]. Zwischen den einzelnen schmalen Cylinderzellen finden sich doppelt und dreifach so breite ungefärbte $Z$ wischenräume. Über diese schrumpfenden Cylinderzellen verlaufen dann noch ein bis zwei Reihen abgeplatteter oberflächlicher Epithellagen, so dass die Zellen vom Charakter der mittleren Reihen eines normalen Hornhautepithels wieder fehlen. Da hier und da einige Hohlräume höher sind als die benachbarten, so ist die Hornhautoberfläche ziemlich uneben.

Genau über der Mitte der Hornhaut findet sich in grösserer' Ausdehnung eine Abhebung der oberflächlichen Plattenepithellagen von der Bowmanschen Membran, welche ron ihnen wie die Sehne rom Bogen überspannt wird. Der Hohlranm zwischen der völlig intakten Bowmanschen Membran und den abgehobenen Epithelien ist leer; nur an den Rändern, wo die Abhebung noeh flacher ist, sieht man einige Zellreste. Das den Hohlraum überspannende Epithelhärutchen zeigt in der Mitte eine kleine Öffnung nach aussen zu (Taf. II, Fig. 8).

In der Conjunctiva bulbi unweit vom Limbus sieht man in den mittleren Epithellagen ebenfalls cystische Hohlränme, die histologisch röllig den beim Cornealepithel beschriebenen gleichen; von letzteren unterscheiden sie sich nur dadurch, dass die Basalzellen nicht in diese Hohlraumbildung einbegriffen sind.

\section{Fall V.}

Frau R., 68 Jahre alt, ist im Verlauf von 2 Jahren völlig auf dem rechten Auge erblindet. Seit einigen Monaten beftige Schmerzanfälle.

Die Hornhant ist rauchig getrübt und zeigt vornehmlich in ihrer unteren Hälfte zahlreiche Unebenheiten in ihrer Oberfläche, bedingt durch feine kleine Bläschen.

Diagnose: Tumor iridis, Status glaucomatosus, Keratitis vesiculosa. 
Enucleation. Aus dem mikroskopischen Bilde sei hier nur mitgeteilt, dass (wie übrigens in geringerem Masse auch bei Fall II) die Epithelkerne sich schlecht färben. Die Epitheldecke ist oben ziemlich unverändert. In der unteren Hälfte ist sie dagegen sehr unregelmässig, stellenweise anf 2-3 Lagen verdünnt, häufiger aber erheblich bis zu 10 und noch mehr Lagen verdickt. Dort, wo das Epithel verdünnt ist, fehlen vielfach in der oberflächlichen Epithelschicht einige Zellen, an deren Stelle dann kleine muldenförmige Defekte sich finden.

Veränderungen ganz anderer Art sieht man dort, wo das Epithel verdickt ist. Hier ziehen mehrfach Kapillaren zwischen Bowmanscher Membran und der Basalzellenlage und auch inmitten des Epithels. Hier zwängen sie sich teils zwischen die neugebildeten polygonalen Zellen, teils auch zwischen die cylindrischen Basalzellen and eine von der Umgebung herïbergewucherte neue Epitheldecke. An mehreren Präparaten lässt sich die Herkunft dieser Gefässe vom unteren Limbus her erkennen.

Die Bowmansche Membran ist überall intakt; man sieht sehr zahlreiche erweiterte Nervenkanälchen sie durchsetzen.

Das vordere Parenchym der Hornhaut zeigt die oft beschriebenen Folgen des Ödems.

\section{Fall VI.}

Michael J., 50 Jabre alt, wird am 3. I. 1907 in die Bonner Augenklinik aufgenommen. Das rechte Auge soll im Jahre 1893 infolge einer Verletzung erblindet sein. Nachdem das Auge im Jahre 1901 noch zweimal Kontusionen erlitten hatte, traten häufig wiederkehrende Schmerzanfälle auf, die seit September 1906 immer stärker geworden sind.

Status praes.: R. A. Ausgesprochene ciliare Injektion; die obere Hälfte der Cornea erscheint matt und gestippt. In ihrer unteren Hälfte sieht man vier kleinere Bläschen, zwei mehr temporal, zwei gerade unter der Popillenmitte. Im unteren Hornhautdrittel sieht man eine ziemlich oberflächliche Trübung, eben dort scheint die Iris der Cornea adhärent. Vom nnteren Limbus zieht ein Gefäss fast bis zu den Bläschen unter der Pupillenmitte. Die obere Hälfte der Kammer hat normale Tiefe. Die Iris schlottert oben, die Pupille stellt ein aufrechtes Oval dar, hinter dem eine geschrumpfte Katarakt liegt. Einblick in die Tiefe ist unmöglich. Leichte Drucksteigerung, Schmerzen.

5. I. Die Bläschen sind geplatzt, an ihrer Stelle sieht man grobe Unebenheiten im Epithel. In der Folgezeit schiessen an der gleichen Stelle stets wieder neue Blasen auf, um wenige Tage später wieder zu platzen.

26. I. Blasenbildang unterhalb der Pupille wie bisher. En ucleation.

Der Bulbus wird in Birch-Hirschfelds Gemisch fixiert und vertikal aufgeschnitten. Die nasale Bulbushälfte mit dem kleineren Hornhautteil wird der Celloidin-Trockenmethode unterworfen, die grössere temporale Cornealhälfte in Paraffin eingebettet.

\section{Mikroskopischer Befund.}

Die vordere Kammer ist unten aufgehoben, die Iris unterhalb von der Pupille überall mit der Hornhauthinterfläche verwachsen. Oben besteht periphere vordere Synechie. Der Schlemmsche Kanal ist erhalten, 
aber vielfach von Rundzellenanhäufungen umgeben. Die Retina ist total atrophisch. In der Chorioidea hat ausgedehnte Knochenbildung stattgefunden; in der unteren Bulbushälfte liegt der Sklera eine Knochenschale von $3 \mathrm{~mm}$ Dicke auf.

Die Cornealgrundsubstanz ist bis auf einige kleine Rundzellansammlungen nahe am unteren Limbus frei von entzündlicher Infiltration.

Die Bowmansche Membran ist überall völlig intakt.

Sowohl am oberen wie am unteren Limbus sieht man zwischen Bowmanscher Membran und Epithel einige Rundzellen, die aber nur in der Randzone der Cornea gefunden werden. Das Epithel ist hier in der Randzone auch überall normal; im unteren temporalen Cornealquadranten ist das Epithel von der Bow manschen Membran etwas abgehoben. Dazwischen liegen sehr zahlreiche rote Blutkörperchen; eigentliche Gefässwandungen oder Lumina sind aber nicht zu sehen. Hier weicht das Epithel auch von der Norm ab; es besitzt nämlich etwa 7-8 Reihen, wobei aber die basale Cylinderzeilenreihe fehlt. Mehrfach sendet das Epithel aus den basalen Zellreihen entspringende zapfen- und zungenartige Fortsätze in den subepithelialen, von Blutkörperchen erfüllten Hoblraum.

Die eigentlich charakteristischen Epithelveränderungen sind auf die Zone der Cornea unterhalb der Pupille beschränkt, also auf die Partie, in der auch klinisch Blasenbildung beobachtet worden war. Nähert man sich dieser Zone sowohl von oben wie von unten, so bemerkt man zunächst eine Zunahme der Epithelreihen und zwar findet man eine basale Reihe von stark abgeplatteten Zellen, 7-8, ja noch mebr Reihen der Stachel- und Riffzellen und wieder eine oberfächliche Lage abgeplatteter Zellen. In den mittleren Lagen dieser Zellschicht findet man hier und da, regellos zerstreut

1. runde Zellen von der normalen Grösse der umgebenden Epithelzellen mit kleinem, aber sehr kräftig tingiertem Kern und schmaler, ihn umgebender protoplasmatiseher Zone, welche wieder ringsum oder mondsichelartig von einer stark lichtbrechenden ungefärbten Zone umgeben wird. Die kleinen Kerne färben sich teilweise bei Weigerts Fibrinfärbung dunkelblau.

2. Viel zahlreicher als diese Zellform findet man eine zweite; diese Zellen haben ebenfalls teilweise die Grösse der umgebenden unveränderten Epithelien, teilweise sind sie auch etwas grösser; sie enthalten entweder einen nur sehr schwach gefärbten Kern, oder lassen überhaupt einen Kern nicht erkennen, haben vielmehr nur einen protoplasmatischen, wenig oder gar nicht gekörnten Zelleib, dessen Peripherie wieder eine stark lichtbrechende, ungefärbte Zone einnimmt (Taf II, Fig. 9a).

3. Weiter sieht man stark - bis auf das drei- und vierfache VoIumen einer normalen Epithelzelle - vergrösserte, aufgequollene, bei den gewöhnlichen Färbemethoden ungefärbt bleibende Zellen, die lebhaft lichtbrechend, fast durchweg ganz strukturlos, homogen erscheinen, nur ganz vereinzelt einige feine dunklere Körnchen erkennen lassen. Diese Zellen färben sich nach Weigerts Fibrinfärbung dunkelblau, bei Thioninfärbung nehmen die gleichen Gebilde den für Schleim charakteristischen, rotvioletten Farbton an, nach $R$ ussel färben sie sich nicht (Taf. II, Fig. 9b). 
4. Ganz vereinzelt endlich findet man auch rundliche bis ovale Gebilde von der Grösse von etwa 5-10 Epithelien, welche gegen die Umgebung durch einen stark lichtbrechenden Saum abgesetzt sind. An einer Seite liegt dann eine Zelle mit Kern und langgestrecktem Protoplasmaleib; den Hauptinhalt aber bildet eine geschlängelte Masse, die Hyalinreaktion gibt: lebhafte Färbung mit Eosin und nach Russel.

Hiermit ist die Fulle der dicht gedrängt nebeneinander vorkommenden Degenerationsformen im Epithel noch nicht erschöpft; man begegnet vielmehr auch noch grossen, aus $30-50$ Zellen hervorgegangenen Hohlräumen im Epithel, die teils ringsum von normalen Zellen umgeben, teils auch oberflächlich gelegen sind; in letzterem Falle ist mehrfach die vordere Wand geplatzt, so dass der Hohlraum frei gegen die Oberfläche zutage liegt.

All diese Epithelveränderungen spielen sich in eiver Zone $a b$, in der zugleich lebhafte Epithelregeneration stattfindet. Mehrfach sieht man nämlich hier zwischen einer mehrreihigen, der Bowmanschen Membran unmittelbar aufliegenden Epithellage und einer oberflächlichen Lage schmale Spalträume, die von dem von allen Seiten über den Spalt heruberwuchernden Epithel in der Mitte noch nicht gedeckt sind.

Diese nenwuchernde Epithelzone ist vornehmlich der Sitz der oben besehriebenen Epithelveränderungen.

Ubbersehen wir nun das Gesamtresultat der genauen mikroskopischen Untersuchung unserer sechs Fälle von Keratitis bullosa (bzw. vesiculosa), so wäre zunächst nochmals darauf hinzuweisen, dass dreimal (Fall I-III) zugleich Pannusbildung nachgewiesen worden ist, einmal (Fall V) wenigstens als erste Andeutung eines ähnlichen Vorganges spärliche Gefässentwicklung inmitten des Epithels beobachtet wurde, während bei den andern Fällen (IV und VI) auch nur die Andeutung einer pannösen Schicht völlig fehlt; um so ausgesprochener sind hier die degenerativen Prozesse mannigfacher Art im Epithel, die auf zwei Momente zurückgefuhrt werden dürfen: auf eine übermässige Durchtränkung des Epithels mit Flüssigkeit und auf eine spezifische Tätigkeit der degenerierenden Zellen (Fall VI); eine Abhebung der Epitheldecke von der Bowmanschen Membran durch eine Flüssigkeitsschicht, die aus dem Cornealparenchym stammt und dann gerinnt, ist nirgends wahrzunehmen.

Überblicken wir nun die Entwicklung der anatomischen Kenntnisse von der Keratitis bullosa, so fällt auf, dass bis in die jüngste Zeit sich zwei Ansichten gegenüberstehen. Wenn wir von den älteren Befunden v. Graefes (34), Schweiggers (35), Saemischs(5) absehen, die alle nur abgetragene Blasenwand untersuchten - ein Verfahren, welches eine einwandsfreie Feststellung des anatomischen Substrates derBlasenbildung nicht ermöglicht —, so hat Klein sch midt (36) 
unter Saemischs Leitung zuerst eine ganze Kaninchencornea mit experimentell erzeugter Blasenbildung untersucht; er fand eine ausgedehnte Abhebung des Epithelblattes und einen mit Flüssigkeit angefüllten Raum zwischen Epithel und der uneben gewordenen vorderen Begrenzungsschicht der Cornea. Fuchs (3) lagen sodann als Erstem Präparate von ganzen menschlichen Augen mit Blasenbildung auf der Hornhaut vor. Er ist der Begründer der Anschauung, dass die Blasen durch einfache Abhebung des Epithels von der Bowmanschen Membran durch eine zwischen beide eingedrungene Ödemflüssigkeit sei. An der Bildung der vorderen Blasenwand kann sich nach Fuchs aber auch eine neugebildete, vom Limbus her entwickelte Bindegewebslage beteiligen.

Traten in der Folge Tartuferi (29) und Bock(30) für Kleinschmidts und Fuchs' (3) Befunde an der Hand von eigenen Untersuchungen ein, so hebt Brugger(31) in seiner unter Eversbuschs Leitung verfassten Dissertation nachdrücklich hervor, dass unmöglich die Blasenbildung auf einer einfachen Abdrängung der Epithelschicht durch seröse Flüssigkeit von der Bowmanschen Membran beruhen könne; vielmehr komme es bei behindertem Flïssigkeitsabfluss infolge Überernährung der Epithelschicht zu reichlicher Neubildung und Wucherung wenig lebensfähiger Zellen, die zum Zerfall neigen. Erst nach Schädigung der Bowmanschen Membran dringt die Stauflüssigkeit vor und führt nun zur Abhebung der Epithelien. Somit sind nach Brugger „die kleinsten Lücken bis zu den kleineren Bläschen von vielleicht Hirsekorngrösse Folge des Zerfalls der Epithelzellen, nicht aber Folge der Quellung und Erweiterung der Intercellularränme durch das Ödem der Hornhaut; die mittelgrossen aber und die grösseren verdanken ihre Entstehung ebenso sehr dem Zerfall von Zellen im Epithel als auch dem gesteigerten Flüssigkeitsgehalte, dem Ödem der Cornea".

Einen weiteren eigenartigen Befund verdanken wir erst wieder Hess (37); er sah in drei Fällen von Blasenbildung bei rezidivierender Erosion, nach Ulcus serpens und bei Glaukoma absolutum die Bläschenwand regelmässig aus Epithel zusammengesetzt, und zwar fand er in grosser Menge mächtige Zellen mit hyalinen Einschliissen, die sich bald als einfache Schollen, bald als lange gewundene keulenförmige Gebilde darstellten; weiter beobachtete Hess auch Vakuolenbildungen neben den Kernen, in einem Falle auch durch amitotische Vermehrung gebildete Kernhaufen. Zur gleichen Zeit beschrieb Nuel(38) eine schleimige und hyaline Degeneration der mittleren 
Epithelzellenlagen an Augen, die matte Oberfläche oder auch Bläschenbildung gezeigt hatten. $\mathrm{Ob}$ hier aber wirklich eine schleimige oder hyaline Umwandlung des Protoplasmas vorgelegen hat, scheint, wie schon Klebs (44) hervorhebt, sehr fraglich, zum mindesten aber nicht sichergestellt, da Nuel keine Mitteilungen über das chemische und färberische Verhalten dieser Form von Epitheldegeneration macht, wie er sie an anderer Stelle (39) für die Keratitis filamentosa gibt.

Hatte Brugger nun schon die Bowmansche Membran an vielen Stellen durchlöchert gefunden, so dass sie gleichsam ein vielfach durchbohrtes, mit ganz unregelmässigen, ausgefressenen Rändern versehenes Sieb darstellte - Veränderungen, welche von Brugger aber als sekundäre angesprochen werden -, so bringt Ewing(33) ein neues Moment in die Frage der Histogenese der Keratitis bullosa. Sie soll nämlich mit einer fettigen Degeneration der Bowmanschen Membran beginnen; im ferneren Verlauf soll es dann zu weiterer Degeneration der vorderen Grenzlamelle mit gleichzeitigem Ergriffensein der anliegenden Schicht der Substantia propria und Absorption der basalen Epithelzellen kommen. Bildung einer Wand für die Blase unter Zunahme der Epithelzellen und schliesslich das Auftreten von Fibrin, Wanderzellen, Spindelzellen und Blutgefässen in der Blase sollen den Prozess abschliessen.

Die letzten von de Schweinitz und Shumway(27) sowie von Wirths (26) herrührenden Untersuchungen bringen keine newen Ergebnisse, vielmehr Bestätigung früherer Befunde. Erstere sahen die Blasenwand aus Epithel zusammengesetzt und beobachteten Zellvermehrung und Vakuolisation um die Kerne. Wirths' Untersuchungen bestätigen im wesentlichen die Befunde von Fuchs (3), „dass nämlich die im Verlaufe von. Glankom auftretenden Blasen durch $\mathrm{Ab}$ hebung von Epithelzellen oder der ganzen Epithelschicht infolge Druckes von Ödemflüssigkeit entstehen, und dass die Wand der kleineren Blasen aus Epithel allein, die der grösseren aus Epithel und einer vom Limbus herkommenden pannösen Bindegewebsschicht besteht". Nach Vossius (51) endlich nimmt an der Bildung der Blasen gewöhnlich nur das Epithel teil.

Zahlreiche Befunde tun also dar, dass die Blasenbildung nicht immer und allein durch einfache Abhebung des Epithels bedingt sein kann, dass vielmehr degenerative Prozesse mannigfacher Art im Epithel zum mindesten ebenso häufig gefunden werden. Dieser Tatsache wird auch ron Fuchs(40) in seiner jüngsten Darstellung des Gegenstandes Rechnung getragen, indem er hervorhebt, dass die 
Trübung der Cornea beim Glaukom nicht allein mehr durch die Anwesenheit abnormer Flüssigkeit zwischen den Zellen hervorgerufen wird, dass vielmehr die Flüssigkeit in die Zellen selbst eindringe und sie aufquellen lässt.

Gleichwohl wird bei der Darstellung der pathologischen Anatomie der Cornea von Greeff(20) als Grundlage der Keratitis bullosa eine Abhebung des Epithels von der Bowmanschen Membran durch Ödem angegeben, und Gama Pinto(41) führt in der französischen Encyklopädie die Entwicklung der Blasen in glaukomatösen Augen auf eine Flüssigkeitsansammlung zwischen der Bow manschen Membran und einer pannösen Schicht zurïck.

Sehen wir nun zu, welche dieser Anschauungen durch unsere Befunde eine Stïtze erfährt und welche nicht, so soll zunächst betont werden, dass der Beginn der Keratitis bullosa bestimmt nicht in einer Degeneration der Bowmanschen Membran gesehen werden kann. Denn unter unsern sechs Fällen, die sämtlich weiter vorgerückte Stadien zeigen, wurde fünfmal die vordere Grenzlamelle völlig intakt befunden. Nur bei Fall I begegnen wir an umschriebener Stelle über einem Hornhautinfiltrat einem Schwund der Bow manschen Membran, der als Folge tieferer entzündlicher Prozesse aufgefasst werden durfte. Dieser Befund entspricht völlig einem gleichen, den de Schweinitz und Shumway (27) in ihrem zweiten Fall erhoben haben; auch hier war die Bowmansche Membran nur über einer alten Ulceration im Parenchym zerstört. Auch Brugger (31) sieht die vielfachen Unterbrechungen der Bowmanschen Membran als ein im späteren Verlauf des anatomischen Prozesses auftretendes Ereignis an, wobei er es unentschieden lässt, ob ein Zerfasern der Membran infolge des Ödems von den Nervenkanälchen aus, oder Atrophie infolge des Druckes oder endlich Resorption die Ursache sei.

Jedenfalls stellt eine fettige Entartung [Ewing (33)] oder irgend welche Entartung anderer Art der Bowmanschen Membrau keineswegs den Beginn der typischen Keratitis bullosa dar. Falls ausgedehntere Zerstörungen oder auch Degenerationen an der vorderen Grenzlamelle auftreten, so werden diese wohl stets als Begleiterscheinung oder Folge tieferer Veränderungen aufzufassen sein, wie sie sich zum Beispiel bei Bandkeratitis und hyaliner Degeneration finden.

Von der Mehrzahl der Autoren wird denn auch die ödematöse Durchtränkung der Cornea, die auch klinisch häufig das Vorstadium. zu der eigentlichen Blasenbildung abgibt, als Ursache dieser letzteren angesehen. Mit dieser Anschauung lassen sich auch unsere Befunde 
wohl in Einklang bringen, wenngleich der Vorgang der Blasenbildung im Epithel sich, wie ich glaube, etwas anders abspielt, als bisher meist angenommen wird. Leber(1) und Fuchs (3) führten bekanntlich zuerst das matte Aussehen glaukomatöser Hornhäute auf die Ansammlung kleinster Tröpfchen $\mathrm{z}$ w is chen den Epithelien der tiefsten Schicht zurïck. Die Flüssigkeit kann die Basalzellen auch ganz von der Bowmanschen Membran abheben oder auch aufwärts in die mittleren Schichten des Epithels vordringen; auch in der obersten Schicht fanden Leber und Fuchs blasenartige Hohlräume. Beide Autoren halten dieselben aber für Flüssigkeitsansammlung zwischen, nicht innerhalb der Zellen und zwar dringt die Ödemflüssigkeit durch die erweiterten Nervenkanäle der Bowmanschen Membran vor, was in der Folge auch Tartuferi (23), Bock(30) und Elschnig (42) bestätigten.

Ist somit das Auftreten von Flüssigkeit in den erweiterten Intercellularräumen bei glaukomatösen Hornhäuten einwandfrei nachgewiesen, so erscheint es doch auf Grund unserer Untersuchungen nicht statthaft, die so gewonnenen Ergebnisse auch auf die an solchen Augen zuweilen zu beobachtenden Bläschen (Keratitis bullosa) zu übertragen. Nach solcher Auffassung, wie sie Leber und Fuchs vertreten haben, fiele dem Epithel eine rein passive Rolle zu: „die Flüssigkeit durchsetzt das Epithel in gewisser Ausdehnung, zerwühlt es und bildet in demselben unregelmässig gestaltete Hohlräume, in welche Trümmer der Epithelzellen hineinragen."

Die intercellulare Flüssigkeitsansammlung, welche an unsern Präparaten vorgerückter Stadien nicht oder nur ausnahmsweise beobachtet werden konnte, ist vielmehr nur die Grundlage des sog. "Hornhautödems"; an der Bildung eigentlicher Hohlräume im Epithel (Keratitis vesiculosa und bullosa) nimmt aber das Epithel selbst auch aktiven Anteil. Es kommt infolge der dauernd behinderten Abfuhr der reichlich zugeführten Ödemflüssigkeit zu einer Aufquellung der Epithelien. Ob bei dieser Vergrösserung der Zellen auch Fetttröpfehen in ihnen auftreten, wie Ribbert(43) es für die Zellen des ödematösen Bindegewebes erwähnt, wie Brugger es bei Keratitis bullosa vermutet, konnte wegen ungeeigneter Fixation allerdings nicht festgestellt werden. Das Protoplasma nimmt infolge der Aufquellung einen mehr und mehr homogenen Charakter an und auch der Kern geht zugrunde; schliesslich reisst die stark gedehnte Zellwand ein, der Inhalt tritt meist wohl direkt nach der Oberfäche aus. Durch Konfluieren mehrerer solcher aus Zellzerfall entstandenen Lücken 
kommt es zur Bildung grösserer Hohlräume, in denen nur vereinzelt Zellreste gefunden werden. Vornehmlich gehen die Basalzellen und die der mittleren Lagen in solche Hohlraumbildung auf; doch beteiligt sich stellenweise auch das ganze Epithel daran.

Die Aufquellung der Epithelien infolge Durchtränkung mit Ödemflüssigkeit und ihre Neigung zum Zerfall mit folgender Lückenbildung, allerdings nur in den oberen und mittleren Schichten, ist schon von Brugger beobachtet worden. A. Klebs (44) führt die Entstehung ausgedehnter Hohlräume im Epithel auf mechanische Momente, auf Druck- und Zugwirkung auf die Zellen zurück. Meines Erachtens ohne zwingenden Grund, denn Klebs konnte in den erweiterten Intercellularräumen keine Flüssigkeit nachweisen, welche durch ihr dauerndes Verweilen die Zellenform beeinflussen konnte. Ich glaube vielmehr, dass man für die Entstehung der Hohlräume im Epithel auch bei Klebs (vgl. dessen Abb. 1, 3, 5, 6, 7) eine Degeneration der Epithelien infolge voraufgegangener hydropischer Quellung annehmen kann. Schlägt doch Klebs selbst vor, statt von Ödem von einer cystoiden Degeneration zu sprechen, eine Auffassung, die eine aktive Beteiligung der Epithelien voraussetzt.

Das interstitielle ödem würde somit die Einleitung (rauchige Hornhauttrübung), Höhlenbildung durch Zerfall der Epithelien den Höhepunkt des Prozesses (Keratitis bullosa bzw. vesiculosa) darstellen [vgl. auch Fuchs(40)]; schliesslich kann Pannusentwicklung und Regeneration des Epithels eine anatomische Heilung herbeiführen.

Durch Konfluieren zahlreicher solcher mikroskopischer Hohlräume können endlich grössere schwappende Blasen mit flüssigem Inhalt entstehen, die platzen, ihren Inhalt nach aussen entleeren und sich bei Fortbestehen der Flüssigkeitsstauung durch abermaligen Zerfall neugebildeter Zellen wiederum entwickeln.

Das anatomische Bild der Keratitis bullosa ist aber, auch wie es sich nach unsern Präparaten darstellt, durchaus kein einheitliches. Ich sehe hierbei ab von den innigen Beziehungen, die sich nach vorliegenden Untersuchungen zwischen degenerativem Pannus und bullöser Keratitis ergeben haben. Man findet nämlich, wie schon erwähnt, öfters als Grundlage des Prozesses eine Abhebung der ganzen Epithelschicht durch eine zwischen sie und die Bowmansche Membran gedrungene Flüssigkeit hervorgehoben. Das Epithel zieht dann bogenförmig über die Hohlräume fort (vgl. Taf. II, Fig. 8). 
Doch wie wir schon genötigt waren, die Entstehung der interepithelialen Hohlräume allein durch Erweiterung der Intercellularräume abzulehnen, vielmehr den Zellen eine aktive Tätigkeit zuzuweisen, so ist die Beteiligung der Epithelien auch bei der Entstehung der subepithelialen Hohlräume unverkennbar. Die basalen Zellen, die wir ja im Unterschied zu Brugger und Klebs häufig affiziert fanden, waren an den betreffenden Stellen bis auf wenige Spuren zugrunde gegangen, und die oberflächlichen erhaltenen Zellagen überwölben in leicht konvexem Bogen den Hohlraum. Also sind auch diese subepithelialen Räume nicht allein durch Abdrängung der ganzen Epithelschicht, sondern auch auf Kosten der basalen Zellage entstanden; abermals ein Beweis für die aktive Teilnahme der Epithelien am Prozesse der Höhlenbildung.

Den bisherigen Erörterungen liegen vornehmlich die Fälle I-V zugrunde. Für diese konnte zwar bestimmt nachgewiesen werden, dass Degeneration der zuvor hydropisch aufgequollenen Epithelien zur Hohlraumbildung führt. Über den Chemismus der Umwandlung dieser Zellen konnte aber so gut wie nichts, jedenfalls nichts Positives ermittelt werden.

Einem ganz andern Typus von Veränderungen begegnen wir aber bei Fall VI. Hier werden Zell- und Degenerationsvorgänge beobachtet, die zusammengehalten mit den Resultaten spezifischer Färbemethoden gestatten, sich ein etwas klareres Bild von der Art des Degenerationsvorganges zu machen.

Zunächst ist es unverkennbar, dass der Zellkern am Degenerationsvorgang erheblichen Anteil hat; er verkleinert sich nämlich und färbt sich gleichmässig und ausserordentlich kräftig mit Kernfarbstoffen; einen direkten Übertritt von Kernsubstanzen in das Protoplasma, wie ihn Lenz(45) bei einem eigenartigen Degenerationsprozess der Hornhautepithelien beschrieben hat, konnte ich hingegen nicht beobachten. Ein Teil dieser kleinen Kerne färbt sich auch nach der Weigertschen Fibrinmethode.

Ein zweites Stadium in diesem Prozess stellen nun die, zum Teil vergrösserten, Epithelien dar, die nur sehr schwach färbbare oder gar keine Kerne mehr besitzen. Als Überreste der letzteren sind vielleicht kleine Körnchen zu deuten, die hier und da im Zelleib liegen. Als weitere Stufe im Degenerationsprozesse gegenüber der zuerst erwähnten Zellform dokumentieren sich diese Zellen auch dadurch, dass eine bei jenen nur in sehr geringer Menge vorhandene stark lichtbrechende ungefärbte Substanz in der Peripherie des Proto- 
plasmas hier schon in reichlicherer Menge, in grösserer Ausdehnung vorhanden ist (Taf. II, Fig. 9a).

Das Endstadium bilden schliesslich die ganz grossen, homogenen, aufgequollenen Zellen, die sich sowohl Kernfarbstoffen wie diffus färbenden Farblösungen gegenüber refraktär verhalten, dagegen die für Fibrin und Schleim charakteristischen Farbreaktionen geben. Da Schleim sich bekanntlich auch nach der $W$ eigertschen Fibrinmethode färbt, darf angenommen werden, dass es sich um schleimige Entartung der Zellen handelt. Diese Umwandlung der Zellen erfolgt nicht nach Art der normalen Schleimsekretion durch Auftreten einzelner Schleimtropfen im Zelleib, vielmehr geht nach und nach eine gleichmässige und restlose Umwandlung der ganzen Zelle in Schleim vor sich (Taf. II, Fig. 9b).

Der Nachweis schleimiger Epithelentartung bei Keratitis bullosa stellt einen bisher nicht bekannten Befund dar. Nuel(38) spricht zwar von einer „Dégénérescence muqueuse de l'épithélium cornéen“, doch geht, wie schon erwähnt, aus seinen Ausführungen nicht hervor, dass seine Annahme durch den positiven Ausfall spezifischer Färbemethoden 'gestützt wird. Sehen wir von diesem allerdings als unerlässlich zu bezeichnenden Nachweise ab, so macht die von $\mathrm{Nuel}$ gegebene Beschreibung einer klaren homogenen, den Zelleib schliesslich fast ganz erfüllenden Flüssigkeit sowie seine Abb. 1 es in gewissem Grade wahrscheinlich, dass ihm ein ähnlicher Prozess vorgelegen hat.

Ebenfalls vermisst man bei Nuel die Begründung, wartum er von „hyaliner Degeneration" spricht; er wählt diese Bezeichnung nämlich, ohne über die Natur des Prozesses etwas aussagen zu wollen. $\mathrm{Da}$ heute aber die Bezeichnung "hyalin" nicht gleichbedeutend mit "homogen" gebraucht wird, vielmehr nur solche homogene, durchsichtige, glänzende, farblose Substanzen darunter verstanden werden, die sich mit den sauren Anilinfarben mehr oder weniger intensiv färben, muss die Beobachtung von Hess als die bisher einzige angesehen werden, die den Nachweis echter hyaliner Entartung des Hornhautepithels bei Keratitis bullosa erbringt. Es verdient daher noch angeführt zu werden, dass im gleichen Fall VI neben der schleimigen Entartung auch hyaline Degeneration, allerdings nur an wenigen Zellen, beobachtet worden ist, und zwar handelt es sich natïrlich um das sekretorische, degenerative, intracellular gebildete Hyalin (Lubarsch) $)^{1}$.

I) Ergebnisse der allgemeinen Pathologie 1895. 
Die beschriebene Epitheidegeneration scheint in mehrfacher Beziehung einige Ähnlichkeit mit der von Franke (46) sogenannten ballonierenden Degeneration des Epithels bei herpetischer und dendritischer Keratitis zu besitzen. Ein wesentlicher Unterschied berubt aber darin, dass im Epithel bei Keratitis bullosa, von mir wenigstens, niemals amitotische Kernteilung beobachtet worden ist.

Auch mit diesen spezifischen Degenerationsvorgängen im Epithel ist die Pathologie der Keratitis bullosa noch nicht erschöpft. Vielmehr müssen wir auf Grund der Präparate von Fall $V$ Ewing beipflichten, dass man in den späteren Stadien auch feine Gefässe inmitten des Epithels trifft; zwischen alte und neue Epithellagen haben sich dann vom Limbus her Kapillaren vorgeschoben. So spärlich kann diese Einsprossung sein, alle weiteren bindegewebigen Elemente so völlig fehlen, dass man von einer eigentlichen Pannusbildung, deren ersten Anfang sie wohl darstellt, kaum reden kann.

Fanden wir also als anatomische Grundlage der Keratitis bullosa einmal eine hydropische Quellung und folgenden Zerfall der Epithelien mit intra- und subepithelialer Höhlenbildung, sodann auch spezifische degenerative Prozesse im Epithel, wurde ferner von andern Autoren Abhebung des Epithels durch eine Flüssigkeitsschicht beobachtet, so liegt es auf der Hand, dass die Bezeichnungen "Keratitis bullosa und vesiculosa“, nach dem klinischen Bilde gewählt, dem anatomischen Bilde nicht eigentlich entsprechen. Vielmehr sind es anatomisch degenerative und wohl auch mechanische Vorgänge (Behinderung der Flüssigkeitsabfuhr), die zur Blasenbildung führen, keineswegs echt entziindliche. Dies ist um so weniger auffallend, als auch klinisch die Blasenbildung im Gefolge einer wirklichen Entzündung, eines parenchymatösen Infiltrates (Saemisch) weit seltener auftritt als an glaukomatösen Augen im Stadium der Degeneration.

Wie vorliegende Untersuchungen zeigen, kann aber das anatomische Bild durchaus kein einheitliches genannt werden. Es erscheint daher nicht zweckmässig, neue und längere und doch nicht die Fülle der anatomischen Prozesse umfassende Namen wie bullöse oder cystoide (Klebs) Degeneration des Hornhautepithels einzuführen. Ist es doch nach unsern heutigen Kenntnissen nicht möglich, schon klinisch festzustellen, ob epitheliale Höhlenbildung oder Abhebung einer mehr oder weniger normalen Epithelschicht durch Flüssigkeitserguss vorliegt. An den beschriebenen sechs Augen konnte ich mich. zwar 
nicht davon überzeugen, dass die Abhebung des Epithels durch eine Flüssigkeitsschicht eine grosse Rolle in der Histologie der Keratitis bullosa spiele; jedoch bürgen die Befunde von Fuchs dafür, dass die Grundlage der Blasenbildung auch eine Abhebung einer normalen Epitheldecke und einer neugebildeten subepithelialen Membran von der vorderen Grenzlamelle durch Flïssigkeitsansammlung bilden kann.

So scheint es mir das Richtigste, an der althergebrachten und eingebürgerten Nomenklatur "Keratitis bullosa" festzuhalten. Jedoch muss man im Auge behalten, dass nicht entzündliche, sondern meist degenerative Prozesse im Epithel die anatomische Grundlage der Erkrankung bilden.

Zum Schluss ist es mir eine angenehme Pflicht, meinen verehrten Lehrern, den Herren Geheimrat Saemisch und Eversbusch für das diesen Untersuchungen gewidmete Interesse herzlich zu danken.

\section{Literaturverzeichnis.}

1) Leber, Über die intercellularen Lücken des vorderen Hornhautepithels im normalen und pathologischen Zustande. v. Graef e's Arch.f. Ophth. Bd.XXIV, 1. 1878.

2) Fuchs, Bericht über die 12. Versammlung der ophthalmologischen Gesellschaft. Heidelberg 1879. Keratitis bullosa. S. 212.

3) - Über die Trübung der Hornhaut bei Glaukom. r. Graefe's Arch. f. Ophth. Bd. XXVII, 3. 1881.

4) Erdmann, Über experimentelles Glankom nebst Untersuchungen am glaukomatösen Tierauge, v. Graefe's Arch. f. Ophth. Bd. LXVI. 1907.

5) Saemisch in Graefe-Saemisch, Handbuch der gesamten Augenheilkunde. I. Aufl. Bd. IV. Krankheiten der Cornea. S. 272.

6) Fuchs, Über Randsklerose und Randatrophie der Hornhaut. v. Graefe's Arch. f. Ophth. Bd. LII. 1902.

7) Müller, H., Würzburger Verhandlungen. Sitzungsber. f. 1855. 7. Sitzung cit. nach 9.

8) Donders, Oederzoekingen etc. cit. nach 9.

9) Alth of, Auflagerungen anf die Lamina elastica anterior. Y. Graefe's Arch. f. Ophth. Bd. VIII, 1. 1862

10) I wan off, Beitrag zur pathologischen Anatomie des Hornhaut- und Linsenepithels. Klin. Beobachtungen aus der Augenheilanstalt zu Wiesbaden. II. 1866.

11) Pagenstecher a. Genth, Atlas d. path. Anat. d. Augapfels. Wiesbaden 1875.

12) Baas, Zur Anatomie und Pathogenese des Pannus und der Phlylktäne. Klin. Monatsbl. f. Augenheilk. 1898 u. 1900.

13) Hertel, Anatomische Untersuchung eines Falles von phlyktänulärer Keratoconjunctivitis. v. Graefe's Arch. f. Ophth. Bd. XLVI. 1898.

14) Augstein, Gefässstudien an der Hornhant und Iris. Zeitschr. f. Augenheilk. Bd. VIII. 1902.

15) Seo u. Yamaguchi, Pathologisch-anatomische Untersuchung von Keratitis faseicularis und Pannus scrophulosus. Klin. Monatsbl. f. Augenheilk. Bd. I. 41. Jahrg. 1908.

16) Ritter, Zur pathologischen Anatomie des Pannus. v. Graefe's Arch. f. Ophth. Bd. IV, 1. 1858. 
17) Raehlmann, Über den histologischen Ban des trachomatösen Pannus. v. Graefe's Arch. f. Ophth. Bd. XXIII, 3. 1887.

18) Michel, Lehrbuch der Augenheilkunde.

19) Junius, Die pathologische Anatomie der Conjunctivitis granulosa nach neuen Untersuchungen. Zeitschr. f. Augenheilk. Bd. VIII. 1902.

20) Greeff, Die pathologische Anatomie des Auges in Orths Lehrbuch der speziellen pathologischen Anatomie. 1902.

21) Fuchs, Lehrbuch der Augenheilkunde. 11. Aufl. S. 99. 1907.

22) Bietti, Über Histologie des Pannus cornealis trachomatosus. Klin. Monatsbl. f. Augenheilk. 41. Jahrg. 1903. Festschr. f. Manz.

23) de Vincentiis, Bericht über den 16. Kongress der italienischen ophthalmologischen Gesellschaft. Klin. Monatsbl. f. Augenheilk. 41. Jahrg. Bd. I. S. 320.1908 .

24) Ginsberg, Grundriss der pathologischen Histologie des Auges. Berlin, Karger 1903.

25) Foster, Über Infiltrationsödem (gallertigen Pannus degenerativus) unter der Bowmanschen Membran. Klin. Monatsbl. f. Augenheilk. 42. Jahrg. Bd. I. 1904.

26) Wirths, M., Beiträge zur Anatomie der Keratitis vesiculosa u. bullosa. Zeitschr. f. Augenheilk. Bd. XVI. 1906.

27) de Schweinitz u. Shumway, Über die Histologie der Keratitis bullosa bei glaukomatösen Augen. Arch. f. Augenheilk. Engl. Ausgabe. Bd. XXXII. Cit. nach der abgekürzten Übersetzung in der deutschen Ausgabe. Bd. LII. 1905.

28) ArJt, Klinische Darstellung der Krankheiten des Auges. Wien 1881. S. 125.

29) Tartuferi, Sull anatomia pathologica della cornea nel glaucoma. Giorn. d. R. Acad. de Anat. di Torino Annales 45, cit. nach Nagels Jahresbericht 1882.

30) Bock, Beitrag zur pathologischen Anatomie der Cornea. Klin. Monatsbl. f. Augenheilk. 1886 .

31) Brugger, Ein anatomischer Beitrag zur Lehre von der Keratitis bullosa. Inang.-Diss. München 1886.

32) Bossalino, Beitrag zur Lehre der pathologischen Anatomie der Hornhaut. Klin. Monatsbl. f. Augenheilk. 1895.

33) Ewing, Bullous Keratitis: Fatty degeneration of Bowman's membrane. Amer. Journ. of Ophth. 1904, cit. nach dem Literaturbericht im Arch. f. Augenheilk.

34) v. Graefe, Notiz über Bläschenbildung auf der Hornhaut. v. Graefe's Arch. f. Ophth. Bd. II. 1855.

35) Schweigger, Handbuch d. Augenheilk. Berlin 1873. S. 322.

36) Kleinschmidt, Utber Keratitis bullosa. Inang.-Diss. Bonn 1876.

37) Hess, Klinische und anatomische Studien über Fädchenkeratitis und einige verwandte Hornhauterkrankungen. v. Graefe's Arch. f. Ophth. Bd. XXXIX. 1893.

38) Nuel, Dégénérescence hyaline et muqueuse de l'épithelium cornéen. Arch. d'opht. T. XIII. 1893, p. 608.

39) - La kératite filamentaire. 2. Communication. Arch. d'opht. T. XIII. 1893. p. 201.

40) Fuchs, On Keratitis. The Bowman Lecture. Transactions of the Ophthalmological Society. Vol. XXII. 1902.

41) da Gama Pinto, Glaucome in Lagrange et Valude Encyclopédie Française d'ophtalmologie. Tome $\bar{V}$. 1906. p. 96.

42) Elschnig, Anatomische Untersuchung zweier Fälle von akutem Glaukom. Arch. f. Augenheilks. Bd. XXXIII. Ergänzungsheft 1896.

43) Ribbert, Lehrbuch d. pathologischen Histologie. II. Aufl. Bonn 1901.

44) Kl ebs, A., Über ödematöse Veränderungen des vorderen Hornhautepithels. Zieglers Beiträge zur pathologischen Anatomie. Bd. XVII. 1895.

45) Lenz, Über eine bisher nicht beschriebene Form von Degeneration des Hornhantepithels. Klin. Monatsbl. f. Augenheilk. 45. Jahrg. Bd. II. 1907.

46) Franke, Über Erkrankungen des Epithels der Hornhaut. Klin. Monatsbl. f. Augenheilk, 44. Jahrg. Bd. I. 1906. 
47) Lott, Über den feineren Bau und die physiologische Regeneration der geschichteten Plattenepithelien. Untersuchungen aus dem Institut für Physiologie und Histologie. Graz 1873. Heft III.

48) Ushiku bo, Bericht tiber Nippon Gangagakkai. Klin. Monatsbl. f. Augenheilk. 44. Jahrg. Bd, II. S. 346. 1906.

49) Meller, Die Sklerose der Cornea. Klin. Monatsbl. f. Augenheilk. 43. Jahrg. Bd. II. 1905.

50) E. v. Hippel, Die Ergebnisse meiner Fluoresceinmethode usw. v. Graefe's Arch. f. Ophth. Bd. LIV. 1902.

51) Vossius, Lehrbuch der Augenheilkunde, 3. Anfl. 1898.

\section{Erklärung der Abbildungen auf Taf. I u. II, Fig. 1-9.}

Fall I. Fig. 1-5. Färbung Hämatoxylin-Eosin.

Fig. 1. Schnitt durch das temporale Hornhautdrittel. $P=$ pannöse Schicht, in ihren oberflächlichen Lagen zellarm. $B=$ Bow man sche Membran. 200 fache Vergrösserung.

Fig. 2. Schnitt durch die Mitte der Hornhaut. $P=$ pannöse Schicht, homogen und zellfrei; wenige Zellen nur zwischen ihr und $B$, der B ow manschen Membran. 200 fache Vergrösserung.

Fig. 3. Übersichtsbild über die Veränderungen etwa über dem temporalen Pupillenrande. Links (unten) liegt das Epithel der B ow man schen Membran unmittelbar anf; bei $E$ Verdickung und Wucherung der Epitheldecke; bei $J$ intraepitheliales Gewebsband. 20 fache Vergrösserung.

Fig. 4. Die pannöse Schicht $P$ teilt sich in ein intraepitheliales, zellhaltiges Gewebsband $J$ und ein subepitheliales Band. $s$. 200 fache Vergrösserung.

Fig. 5. Intraepitheliales, zellfreies homogenes Gewebsband $J . B=$ Bowmansche Membran. 200fache Vergrösserung.

Fall II. Fig. 6-7. Färbung Heidenhain-van Gieson.

Fig. 6. Höhlenbildung im Epithel durch Untergang von Epithelzellen. 200 fache Vergrösserung.

Fig. 7. $P=$ lockere pannöse Schicht; sie wuchert noch in die Randzone der Epitheldegeneration ein, $B=$ Bow man sche Membran. 200 fache Vergrösserung.

Fall IV. Fig. 8. Hohlraum über der Bowmanschen Membran, entstanden durch Untergang der tieferen und Abhebung der oberflächlichen Zellagen. Am Rande des Hohlraums einige Zelltrümmer; kleine zentrale Öffnung. 100fache Vergrösserung.

Fall VI. Fig. 9. Färbung Hämatoxylin-Eosin.

$a$ aufquellende Epithelien teils ohne, teils mit nur schwach gefärbtem Kern; das Protoplasma wird von einer ungefärbten, stark lichtbrechenden Randzone eingesäumt.

$b$ grosse, anfgequollene, völlig homogene Zellgebilde, die sịch nur bei den Methoden zum Fibrin- und Schleimnachweise färben. Vergrössernng 340 . Zeiss Okular 2. Objektiy E.

Die Abbildungen $1-8$ sind mit dem Zeiss schen mikrophotographischen Apparat der Bonner Universitäts-Augenklinik von meinem lieben Kollegen Dr. Plask uda angefertigt, dem ich auch an dieser Stelle meinen herzlichsten Dank sage. 
हैं

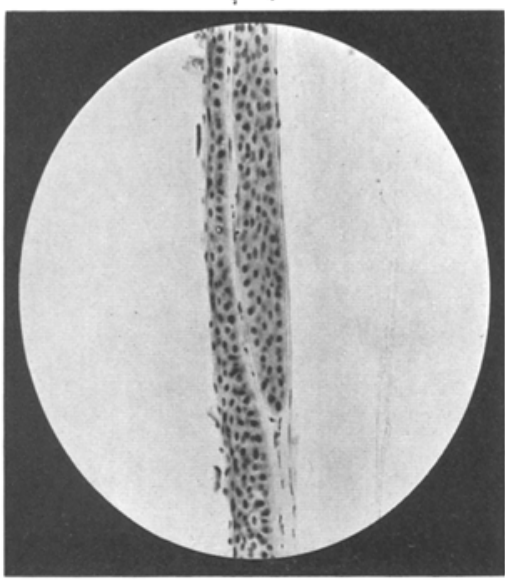

$\dot{x}$

2

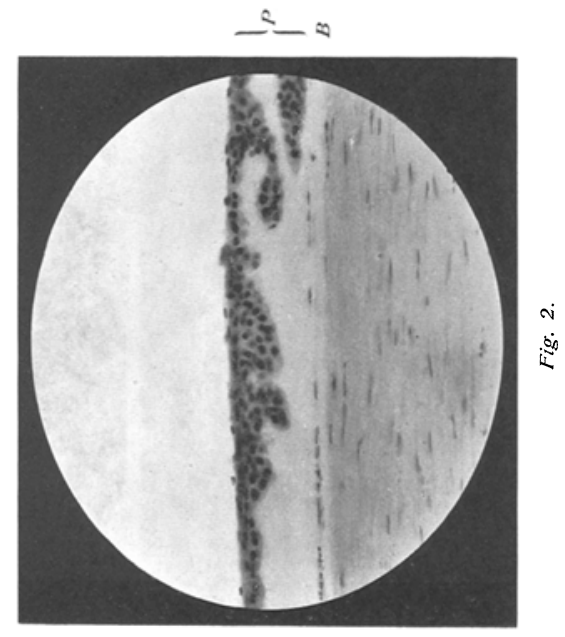

व:

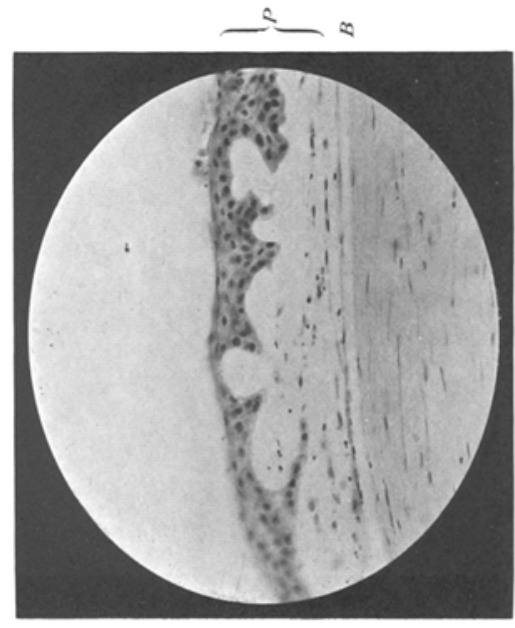

事

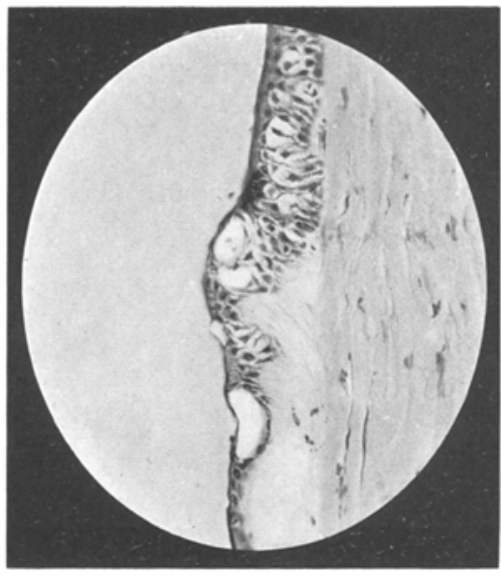

$-\infty \infty$

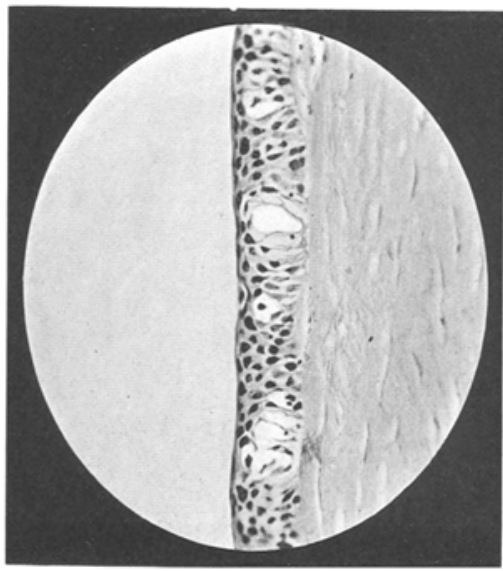

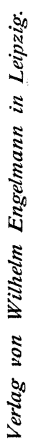

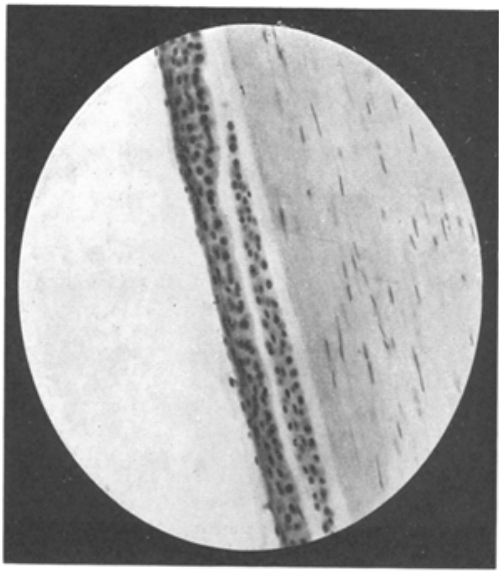

$-\infty$ 


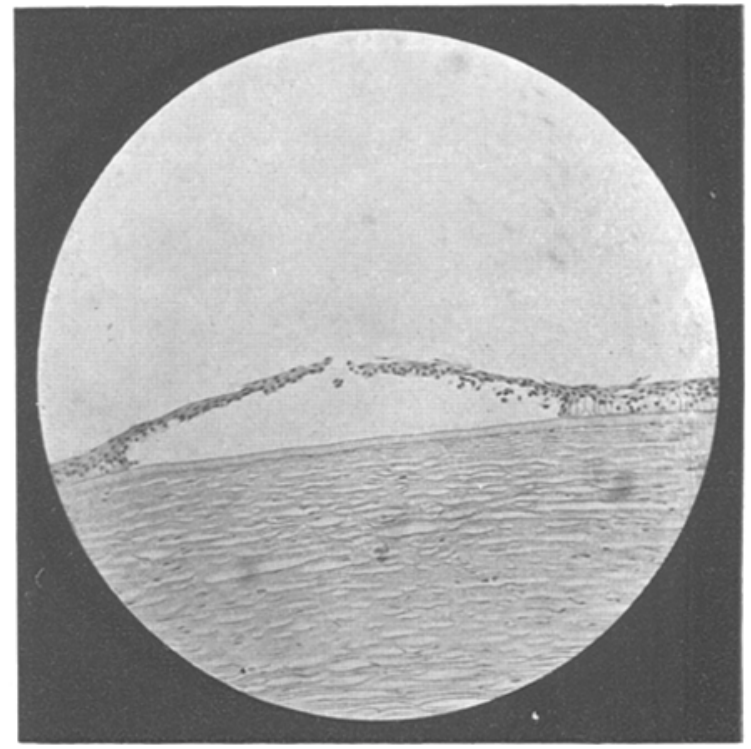

Fig. 8.

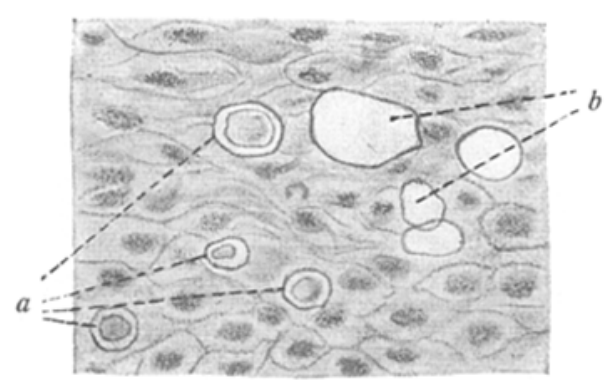

Fig. 9.

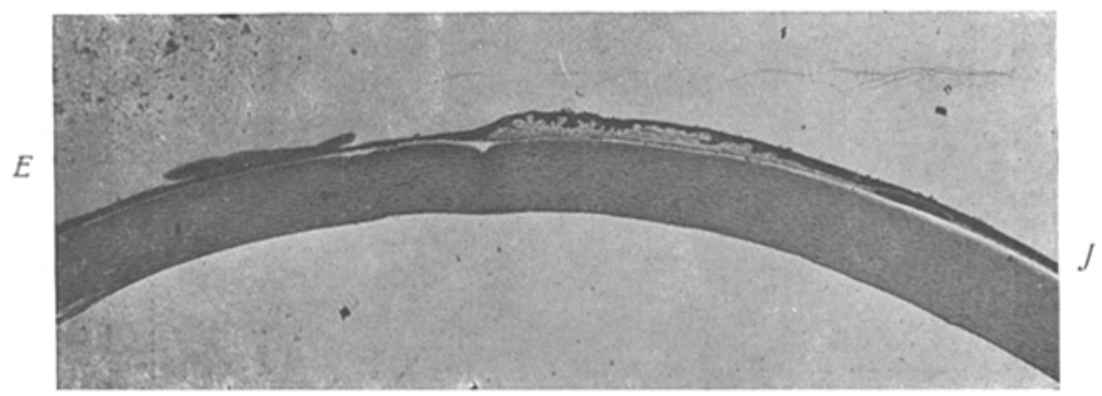

Fig. 3.

Verlag von Withelm Engelmann in Leipzig. 